\begin{tabular}{|l|l|}
\hline 2. To: (Receiving Organization) & 3. From: coriginating Organization) \\
Distribution & DST Retrieval Projects \\
\hline 5. Proj./Prog./Dept./Div.: & 6. Design Authority/ Design Agent/Cog. \\
W-151/Retrieval & Engr.: \\
\hline 8. Originator Remarks: & M. Nordquist \\
\hline
\end{tabular}

This EDT releases the test report for checkout testing on Project $W-151$. This test report documents completed testing of all installed equipment in preparation for the operational test procedure TF-210-0TP-001. Design verification was performed by the informal method.
11. Receiver Remarks:
11A. Design Basel ine Document?
[] Yes
$[X]$ No
None.

4. Related EDT No.:

$$
N / A
$$

7. Purchase Order No.:

$$
\mathrm{N} / \mathrm{A}
$$

9. Equip./Component No.:

$\mathrm{N} / \mathrm{A}$

10. System/Bldg./Facility:

$$
N / A
$$

12. Major Assm. Dwg. No.: $N / A$

13. Permit/Permit Application No.: $\mathrm{N} / \mathrm{A}$

14. Required Response Date: $\mathrm{N} / \mathrm{A}$

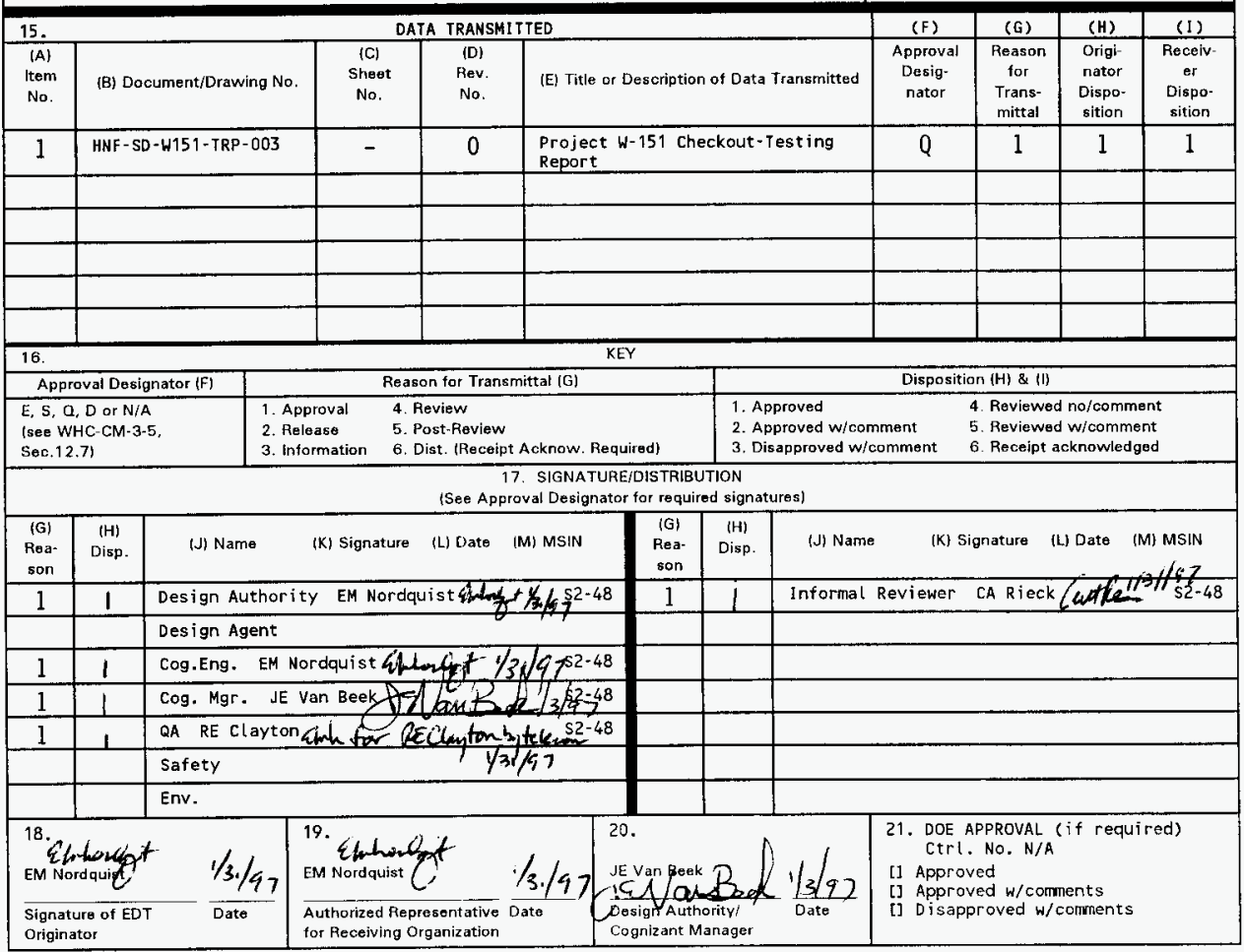




\title{
Project W-151 Checkout-Testing Report
}

\author{
E. M. Nordquist
}

Numatec Hanford Company, Richland, WA 99352

U.S. Department of Energy Contract DE-AC06-96RL13200

$\begin{array}{lll}\text { EDT/ECN: } & 610579 & \text { UC: } 720 \\ \text { Org Code: } & 8 C 430 & \text { Charge Code: D2D1A } \\ \text { B\&R Code: } & 39 E W 31301 & \text { Total Pages: } 4547 \text { cas } 1 / \mathrm{s} / \mathrm{T}\end{array}$

Key Words: Project $W-151$, mixer pumps, 101-AZ, retrieval system, checkout testing, test report

Abstract: This document contains the completed checkout testing plan along with the exception log documenting exceptions which occurred during the test and their closure. This document also contains several minor open exceptions which will be closed upon installation of the radiation-sensitive equipment.

TRADEMARK DISCLAIMER. Reference herein to any specific commercial product, process, or service by trade name, trademark, manufacturer, or otherwise, does not necessarily constitute or imply its endorsement, recommendation, or favoring by the united states Government or any agency thereof or its contractors or subcontractors.

Printed in the United States of America. To obtain copies of this document, contact: WHC/BCS Document Control Services, P.O. BOX 1970, Mailstop H6-08, Richland WA 99352, Phone (509) 372-2420; Fax (509) 376-4989.
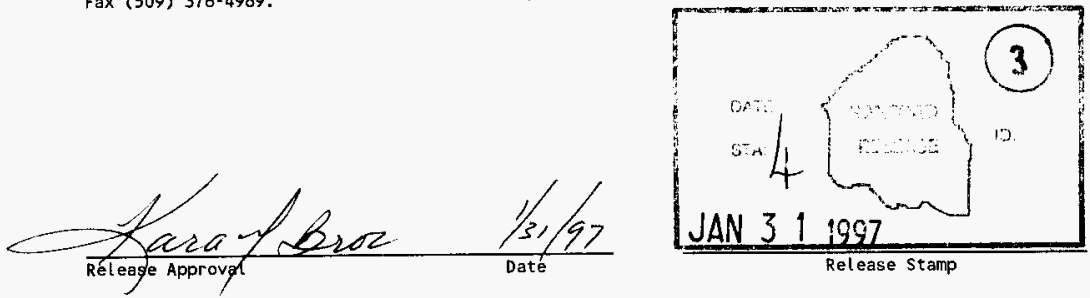

\section{Approved for Public Release}




\section{RECORD OF REVISION}

(2) Title

Project W-151 Checkout-Testing Report

CHANGE CONTROL RECORD

\begin{tabular}{|c|c|c|c|}
\hline \multirow{2}{*}{ (3) Revision } & \multirow{2}{*}{ (4) Description of Change - Replace, Add, and Delete Pages } & \multicolumn{2}{|c|}{ Authorized for Release } \\
\hline & & (5) Cog. Engr. & (6) Cog. Mgr. Date \\
\hline 0 & $\begin{array}{l}\text { (7) Initial release of checkout testing } \\
\text { report (EDT } 610579 \text { ). }\end{array}$ & $\begin{array}{l}\text { EM Nordquist } \\
\text { Elnhout }\end{array}$ & JE Van Beek $\mathrm{Ac} / 3 \mathrm{G}$ \\
\hline & & & \\
\hline & & & \\
\hline & & & \\
\hline & & & \\
\hline & & & \\
\hline & & & \\
\hline & & & \\
\hline & & & \\
\hline & & & \\
\hline & & & 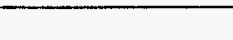 \\
\hline & & & \\
\hline & & & \\
\hline & & & \\
\hline & & & \\
\hline & & & \\
\hline & & & \\
\hline & & & \\
\hline & & & \\
\hline & & & \\
\hline & & & \\
\hline & & & \\
\hline & & & \\
\hline & & & \\
\hline & & & \\
\hline & & & \\
\hline & & & \\
\hline & & & \\
\hline & & & \\
\hline & & & \\
\hline & & & \\
\hline & & & \\
\hline & & & \\
\hline & & & \\
\hline
\end{tabular}




\section{Project W-151 Checkout-Testing Plan}

E. M. Nordquist

Westinghouse Hanford Company, Richland, WA 99352

U.S. Department of Energy Contract DE-ACO5-87RL10930

ECN 2 (15/-30)

EDT/ECN: $612620 \mathrm{kms} \% / / \mathrm{k} \%$ UC: 721

Org Code: 8 K520

B\&R Code: 39EW31301 Total Pages: 44

Key Words: Project $W-151$, mixer pumps, 101-AZ, retrieval system

Abstract: This document contains the steps necessary to document

checkout of the Project W-15l equipment in preparation for the mixer P:-... $r$; ocess test (TF-210-0TP-001).

TRADEMARK DISCLAIMER. Reference herein to any specific comercial product, process, or service by trade name, trademark, manufacturer, or otherwise, does not necessarily constitute or imply its endorsement, recointendetion, or favoring by the United steites Goverment or any agency thereof or its contractors or subcontractors.

Printed in the United States of America. To obtain copies of this document, contact: WHC/BCS Document Control Services, P.0. Box 1970, Mailstop H6-08, Richland WA 99352, Phone (509) 372-2420; Fax (509) $376-4989$.
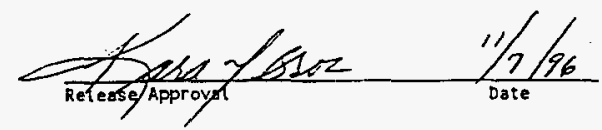

Approved for Public Release

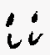




\begin{tabular}{|c|c|c|c|c|}
\hline & RECORD OF REVISION & $\begin{array}{l}\text { (1) Document Nun } \\
\text { WHAC-SD-W151- }\end{array}$ & If-003 & Page $i$ \\
\hline $\begin{array}{l}\text { (2) ritle } \\
\text { Project W-1 } \\
\text { Tank 101-AZ }\end{array}$ & $\begin{array}{l}1 \text { Checkout-Testing } \mathrm{Plan} \\
\text { Waste Retrieval System }\end{array}$ & HNF & RPP ithe & \\
\hline & CHANGE CONTROL RECORD & & & \\
\hline (3) Revision & (4) Deseription of Change - Replace, Add, and Delete Pages & Authori & ed for Re & \\
\hline (2) kevisitit & & (5) Cog. Engr. & (6) $\cos$ & Date \\
\hline 0 & (7) Initial Release via EOT 612620 & EM Nordquist & JE Van $\mathrm{B}$ & \\
\hline OA ES & $\begin{array}{l}\text { Replace pages } 2 \text { through } 13,22,27 \text {, and } 32 \\
\text { via ECN W151-301, correcting eng ineering } \\
\text { omissions and minor editorials. }\end{array}$ & EM Nordquist & JE & Mintor \\
\hline & & & & \\
\hline & & & & \\
\hline & & & & \\
\hline & & & & \\
\hline & & & & \\
\hline & & & & \\
\hline & & & & \\
\hline & & & & \\
\hline & & & & \\
\hline & & & & \\
\hline & & & & \\
\hline & & & & \\
\hline & & & & \\
\hline & & & & \\
\hline & & & & \\
\hline & & & & \\
\hline & & & & \\
\hline & & & & \\
\hline & & & & \\
\hline & & & & \\
\hline & & & & \\
\hline & & & & \\
\hline & & & & \\
\hline & & & & \\
\hline & & & & \\
\hline & & & & \\
\hline & & & & \\
\hline & & & & \\
\hline - & & & & \\
\hline
\end{tabular}


Table of Contents

Execution and Plan Approval ................. 3

1. Purpose ...................... . 4

2. References .................. . . . 4

3. Responsibilities ................. . . 4

4. Change Control ................. 5

5. Execution ..................... 5

6. Exceptions .................... 5

7. Prerequisites................. 5

8. Equipment Checks ................. 6

9. Mixer Pump Rotation Checks .............. 8

10. Mixer Pump Wiring Checks ............... 11

11. Check operation of main Pump 1 drive ........... 11

12. Check operation of main Pump 2 drive .......... 12

Appendix A - Checkout-Testing Exceptions ........... 13

Appendix B - Pump I Rotation Drive Checkiist ........... 14

Appendix C - Pump 2 Rotation Drive Checklist ........... 18

Appendix 0 - Pump 1 Main Drive Checklist ............ 22

Appendix E - Pump 2 Main Orive Checklist ........... 27

Appendix F - Pressure Switch Checklist . . . . . . . . . . 32

Appendix $G$ - Vibraswitch Checklist .............. 33

Appendix $\mathrm{H}$ - Control Console Checklist . . . . . . . . . . . 35

Appendix I - Motor Bearing Temp Checklist . . . . . . . . . . . 36

Appendix $\mathrm{-} \mathrm{Strain} \mathrm{Gage} \mathrm{Checklist} \mathrm{..............} 38$

Appendix K - Sludge Mobilization Cart Checklist . . . . . . . . 40

Appendix $\mathrm{L}$ - Instrument Check List . . . . . . . . . . 41 


\section{Execution and Plan Approval}

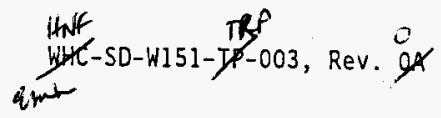

\section{Executed by:}

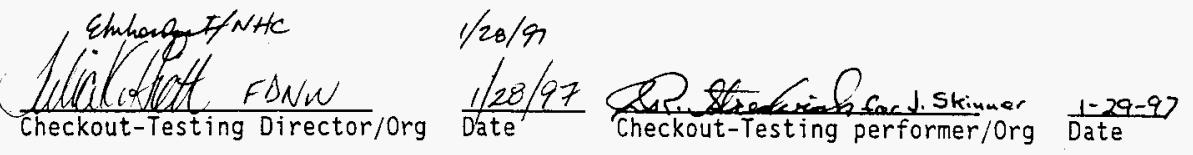

$\overline{\text { Checkout-Testing performer }} / 0$ grg Date $\overline{\text { Checkout-Testing performer }} / 0$ rg $\overline{\text { Date }}$

\section{Witnesses:}
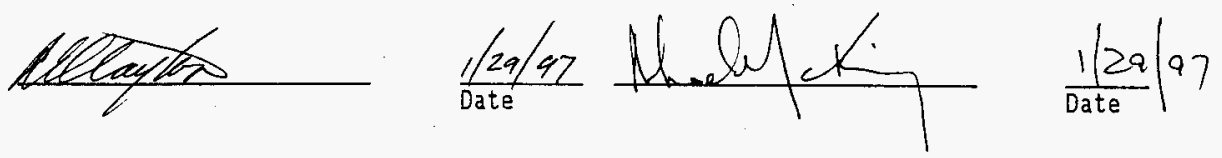

Approval and Acceptance: WITH EXCEPTIONS PER APPENDIX A SHEET 3 A $\xi B$
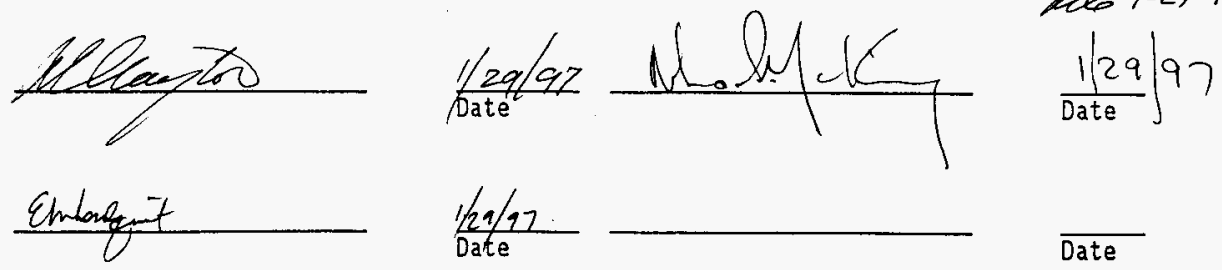

$\overline{\text { Date }}$ 


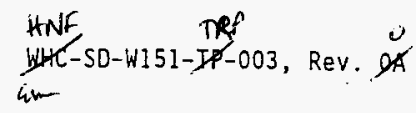

Project $W-151$ Construction Checkout-Testing

1. Purpose

This Construction Checkout-Testing $\mathrm{Plan}$ ( $\mathrm{Pl}$ an) has been prepared to demonstrate and document that the Project $W-151$ equipment has been installed as designed.

2. References

2.1 Drawing List - $\mathrm{H}-2-78870$

2.2 Specifications

2.2.1 HHC-S-0040 Mixer Pump Specification

2.2.2 V-W151-0002 Variable Frequency Drives

2.2.3 V-W151-0004 Sludge Monitoring Cable and Cabie Reel

2.2.4 W-151-C2 Construction Specification (Construction

2.2.5 W-151-C3 Construction Specification (Mixer Pump Control Facility)

2.3 Vendor Information (VI) - VI $\sharp 22515$

2.3.1 Supplement 15 - Variable frequency drives

2.3.2 Supplement 16 - Sludge mobilization monitoring system

2.3.3 Supplement 17 - Control room console

2.3.4 Supplement 20 - Transformer and switchgear

2.3.5 Supplement 32 - Mixer pumps

2.4 Engineering Change Notices (ECN)

Prior to documenting final execution and Plan approval, log ECN numbers written against this Plan. ECN 635012,163866, 635093,

$$
635095=241-2897
$$

3. Responsibilities

3.1 General - Any of the below designated individuals may record items in this plan. These recorded items include the names of participants, instrument identification numbers and expiration dates, each step as it is completed, and any objections and exceptions.

3.2 Project Engineer - responsible for designating Checkout-Testing (C-T) Director, coordinating activities with the facility, and acts as a liajson between participants. 


$$
\text { WW }
$$

3.3 C-T Director - coordinates and directs checkout-testing, ensures the system is left in a safe mode during outages, approves changes to this Plan, may alter $C-T$ sequence (after verifying no adverse impacts), and signs on the final acceptance page.

3.4 C-T performer(s) - performs the actual step using equipment and instruments as necessary (this person may change depending on the craft resource needed for the step, generally expected to be an electrician).

3.5 Witness(es) - observes the $C-T$, reviews the results, and signs on the final acceptance page.

4. Change Control

Required changes to this Plan shall be by the redline method, approved by the C-T Director.

5. Execution

Individuats shall carry out their assigned work in a safe manner per the process control package to protect themselves and others from undue hazards and to prevent damage to property and the environment. Facility line managers shall assure the safety of activities within their areas to prevent injury, property damage, or interruption of operation. Performance of $C-T$ activities shall always include safety and health aspects. C-T step sequence may only be altered by the C-T Director after verifying no adverse impacts.

6. Exceptions

Exceptions to the required $C-T$ results are sequentially numbered and recorded. Errors in the C-T test shall be processed per Section 4, not as $\mathrm{C}-\mathrm{T}$ exceptions. Actions taken to resolve the exceptions shall be documented and approved by the C-T Director. Rechecking of the step(s) shall be performed and documented on the exception form. The exception form is included in Appendix A.

7. Prerequisites

\section{I Conditions}

7.1.1 Systems inspected for compliance with construction documents. 


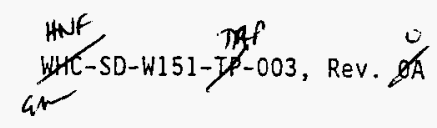

7.1.2 Reference documents verified for latest revision and outstanding ECNs.

7.1.3 Pre-job safety analysis completed and a pre-job safety meeting conducted.

7.1.4 Instruments have a valid calibration stamp attached and instrument identification numbers and calibration expiration dates recorded in 7.2 .

\subsection{Equipment/Instruments}

7.2.1 Volt-ohm-meter (VOM): Digital, portable, $0-1000 \mathrm{~V} \mathrm{ac} / \mathrm{dc}$ Instrument No. 950-45-08-027 Cal Exp Date $4 / 30 / 97$
Instrument No. CaT Exp Date

CaT Exp Date

7.2.2 Test leads with insulated covers for wire clips.

7.2.3 Pressure gauge, analog or digital, range 0-100 psi minimum, accuracy $\pm 2 \mathrm{psi}$. Inst No. 950-31-04-067 Cal Exp Date $8 / 14 / 97$

7.2 .4 Portable DC power supply, 0 to 10 volts.

8. Equipment Checks - The following sections check the installed equipment to verify correct terminal wiring within the individual junction boxes and the various instruments respond correctly when a known signal is applied. All power feeding each piece of equipment shall be locked out per WHC-CM-1-10, WKS 8.2, prior to opening the boxes.

8.1 Check Rotation Drive WST-M-704A - Pump 1 (WST-P-702A)

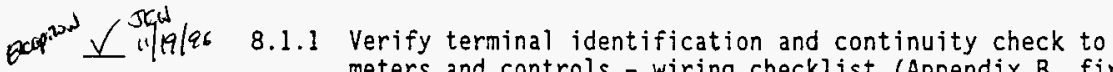
meters and controls - wiring checklist (Appendix B, first page).

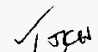

$11 / 1996$

8.1.2 Verify emergency stop - continuity check

8.2 Check Rotation Orive WST-M-703A - Pump 2 (WST-P-701A)

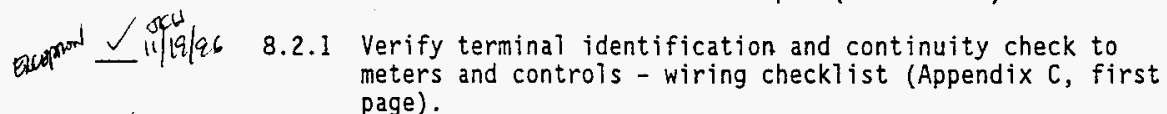
$\underline{V}_{11}|9| 96 \quad 8.2 .2$ Verify emergency stop - continuity check 


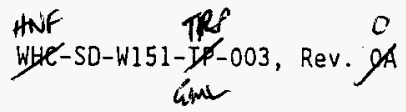

8.3 Check Main Pump Drive - Pump I (WST-P-702A)
expept $\frac{5 C H}{12-13-96}$
8.3.1 Verify terminal identification and continuity check to meters and controls - checklist (Appendix D).
$\frac{\pi+4}{12+3-96}$
8.3.2 Verify start, stop, and emergency stop pushbuttons - continuity check.

8.4 Check Main Pump Drive - Pump 2 (WST-P-701A)
exapin $\frac{\pi \mathrm{CH}^{2}}{12-13-96}$
8.4.1 Verify terminal identification and continuity check to meters and controls - checklist (Appendix E). $\frac{\operatorname{sen} 13-96}{12-13}$
8.4.2 Verify start, stop, and emergency stop pushbuttons - continuity check.

8.5 Check pressure switches

8.5.1 Verify indicator lights in 241-A2-801A and on control console. Note the fights are identical in both $801 \mathrm{~A}$ and on the console; to accept each step the lights must actuate in both places. Valves SW-V-767 and SW-V-770 are closed. Valve $5 W-V-758$ (outside hose line) is also closed. Follow appropriate radiation control procedures for breaking of water ines and disposal of water. If the alarm trips do not occur within the ranges indicated, adjust the trip settings and retest.

$$
\begin{aligned}
& \frac{\pi x+12-96}{12-12} \\
& 2000 \frac{\cos t}{11-21-96} \\
& \text { atedent } \frac{\mathrm{cht}}{11 / 2196}
\end{aligned}
$$

8.5.1.1 Differential pressure (dp) SW-PDIS-750. Verify normal light (green) for 0 psi, and high dp light (red) at 5 psi \pm 1 psi. Record pressure for high dp light trip. 5 psi.

8.5.1.2 Pressure switch SH-PSL-750 - verify normal light (green) at 60 s 1 i and low al arm light (red) at 40 psi ( +0 psi, $-20 \mathrm{psi}$ ). Record pressure for low al arm light (red). 37 psi

8.5.1.3 Pressure switch SWLPSL -751 - verify normal light (green) at $6 \theta^{2} \mathrm{si}^{2}$ and low al arm light (red) at 40 psi (+0 psi, $-20 \mathrm{psi})$. Record pressure for low a larm light (red). 38 psi

8.5.2 Refer to wiring checklist if problems occur (Appendix F).

8.6. Check vibration switches

8.6.1 Verify indication occurs on console screens by backing off the setpoint adjusting screw two turns $\mathrm{CCW}$, press the reset 
button, and turning the setpoint screw $\mathrm{CW}$ slowly until actuation occurs (armature assembly contacts the latch magnet). Confirm lights on console turn on to indicate a vibration problem. Mark final setting on Vibraswitch cases as "zero vibration point." Press reset button.

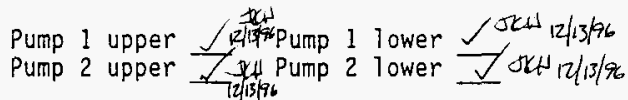

8.6.2 Refer to wiring checklist (Appendix G) if problems occur.

$\sqrt{1 / 797}$

$\sqrt{1 / 17} 197$

8.7 Check computer data acquisition wiring and terminal connections

8.7.1 Control console digital panel meters - wiring checklist (Appendix H).

8.7.2 Motor bearing temperatures - wiring checklist (Appendix I).

8.7.3 Strain gages - wiring checklist (Appendix J).

8.7.4 Sludge mobilization cart data via JB101-100 \& JB101-101 wiring checklists (Appendix K).

8.7.5 Indicator light status - note all lights were checked in steps 8.5 and 8.6 above.

8.8 Checkout signals to monitoring console - use Appendix L, checking for each step identified for each instrument.

9. Mixer Pump Rotation Checks - The following sections check the rotation of the mixer pump assemblies. Breaker $A 3$ in $M C C-702$ is closed and the local disconnect switches DS-703A and DS-704A are closed. Check operation of mixer pump rotation drives \& control console functions. Control console will be plugged in and operational.

9.1 Verify function \& setting for Pump l's rotation motor (WST-M-704A) as follows:

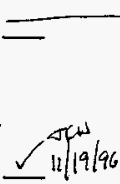

9.1 .1 .

Position-potentiometer, should-read zero when console- is turned on and operable. If not, adjust panel meter scale

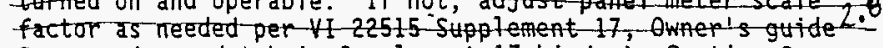

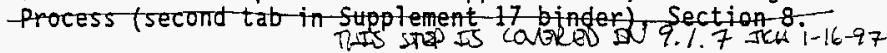

9.1.2 Power up and adjust the variable frequency drive per Instruction Manual 02-3166-7, Sections 4 and 5 (VI 22515 Supplement 15 , ninth tab in binder). Check all VFD parameters and record values in Appendix $B$. 


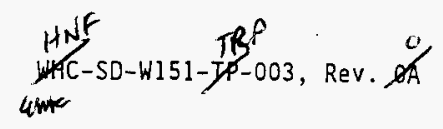

$\mathcal{J} \pi / 19 / 96$

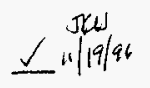

\section{1 .2 .1}

Set VFD deceleration to 1.5 Seconds -- page 5.16 , change function 45 of menu 2 to 1 (enable) first, then change function 2 of menu 1 to 1.5 seconds.

9.1.3 Verify that all VFD parameters and accesses are secured.

9.1.4 Limit switches - turn motor on at control console by pressing start switch. [Note, control switch on console will always be run in the manual setting (manual/automatic) and in the forward setting (forward/reverse).] Speed should be set a min value on control knob on console.

9.1.4-1-Attuate-first-limit switch by hand.-Verffy rotation ehanges to-the other direction. $\mathrm{JKH}_{12-23-96}$

2.1.4.2- Actuate-the second-7imit switeh $190^{\circ}$-out from the -

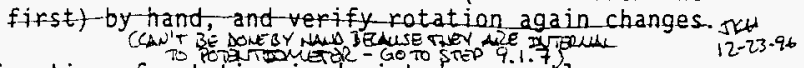

$\sqrt{x+100}$

9.1 .5

Verify direction of rotation is
monitoring console. $\quad \in+\infty \rightarrow+$

9.1.6 Verify emergency stop function stops rotation motor.

9.1.7 Limits switches - Turn motor back on and allow turntable to proceed to first limit switch and verify the limit switch actuates and changes direction at $90^{\circ}$ to $92^{\circ}$ sopeduce VFD ramp-down time if needed. Allow turntable to proceed to the second switch and verify limit switch actuates and

$\underbrace{503}_{1} 16197$

9.1 .8 changes turntable direction at $-90^{\circ}$ to $-92^{\circ}$.

92.2

90.9

700

$-98.2$ $\left.\cos ()^{\prime}\right)$

$\underline{\sqrt{3}}+10.97$ Verify position in
console indication.

field is within $5^{\circ}$ of control verify rotation motor shutdown.

$\sin _{10-47}$

9.1.9 Overtravel -- manually actuate each overtravel switch to

9.1.10 Observe overhead cable behavior -- 6 cycles minimum (either during previous operations or turn motor back on). Note if twisting appears excessive, if bending is smaller than the minimum bend radius for the specific cable $\left(6.64^{*}\right.$ for $4 / 0$ cable and 4.88" for $1 / 0$ grounding cable), any excessive rubbing, and that the cables have enough slack. If problems occur, notify C-T Director and/or open local disconnect (DS-704A). 


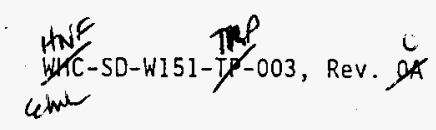

9.2 Verify function \& setting for Pump 2's rotation motor (WST-M-703A) as follows:

9.2.1 Position-potentioneter, shoutd read zero when console is turned-on and operabie. If not, adjust-panel meter scate factor-as needed per. VI 22515 Supplement 17 , Owner's guide Process-(second tab in supptement 17 binder) Section 8 . Thes

$\checkmark \|{ }_{19} / 96 \quad 9.2 .2$ Power up and adjust the variable frequency drive per

Instruction Manual D2-3166-7, Sections 4 and 5 (VI 22515

Supplement 15). Check all VFD parameters and record values in Appendix $C$.

$\checkmark 11 / 19 / 96$

9.2.2.1 Set VFD deceleration to 1.5 Seconds -- page 5.16, change function 45 of menu 2 to 1 (enable) first, then change function 2 of menu 1 to 1.5 seconds.

$\mathcal{L}_{1104}^{\pi / 96}$

9.2.3 Verify that all VFD parameters and accesses are secured.

9.2.4 Limit switches - turn motor on at control console by pressing start switch. [Note, control switch on console will always be run in the manual setting (manual/automatic) and in the forward setting (forward/reverse).] Speed should be set on min value on control knob on console.

9.2.4.1 Actuate first fimit-switch by hand-.-. Verify rotation-changes to the-other-direction, Jow 12-23-96

9.2 4.2- Actuate the second -imit switeh $\left(90^{\circ}\right.$ - $\theta$ ut from the fifst) by hand and verify rotation again-changes- oku

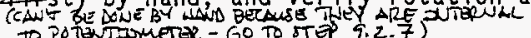

/1164197

$\sqrt{16197}$ $\sqrt{116947}$

$\sqrt{116 \mid 97}$ S1.16197

\section{2 .5}

9.2.6 Verify emergency stop function stops rotation motor.

9.2.7 Limit switches - Turn motor back on and allow turntable to proceed to first 1 imit switch and verify the limit switch actuates and changes direction at $90^{\circ}$ to $92^{\circ}--$ reduce VFD ramp-down time if needed. Allow turntable to proceed to the second switch and verify limit switch actuates and changes turntable direction at $-90^{\circ}$ to $-92^{\circ}$.

9.2.8 Verify position in the field is within $5^{\circ}$ of monitoring console indication.

9.2.9 Overtravel -- manually actuate each overtravel switch to verify rotation motor shutdown. 


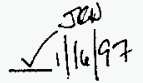

9.2.10 Observe overhead cable behavior -- 6 cycles minimum (either during previous operations or turn motor back on). Note if twisting appears excessive, if bending radius is smaller than the minimum bend radius for the specific cable $(6.64 "$ for $4 / 0$ cable and 4.88 " for $1 / 0$ grounding cable), any excessive rubbing, and that the cables have enough slack. If problems occur, notify $C-T$ Director and/or open local disconnect (DS-703A).

10. Mixer Pump Wiring Checks - Visual and continuity check the motor wiring for both mixer pumps. Note for these steps breakers $B 7$ and $B 8$ in MCC-701 are open and locked out.

10.1 Visual and continuity check Pump 1 motor wiring $-31 / 14 \mid 96$

10.1.1 Perform continuity check of wiring between VFD-702A and Breaker B8, and between VFD and DS-702A.

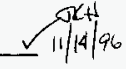

10.1.2 Isolate motor from VFD by opening DS-702A, and megger check motor and wiring.

10.1.3 Verify connections are tight.

10.1.4 Verify pump heater has been turned on - 5 days minimum before motor operation. (Record date $11 / 7 / / 6$ )

10.2 Visual and continuity check Pump 2 motor wiring SCA $\sqrt{11} / 14 / 96$

10.2.1 Perform continuity check of wiring between VFD-701A and Breaker B7, and between VFD and DS-701A.

$\sqrt{11 / 14 / 96}$

10.2.2 Isolate motor from VFD by opening DS-701A, and megger check motor and wiring.

10.2.3 Verify connections are tight.

10.2.4 Verify pump heater has been turned on - 5 days minimum before motor operation. (Record date $1 / 7 / 96)$

11. Check operation of main Pump 1 drive - obsèrve for interference with instrumentation signals (e.g., harmonic interference). Note breaker B8 in MCC-701 is closed, DS-702A is closed, and pump coupler is removed and locked out. (Note, any Vibraswitch lights turning on should be ignored as the final adjustment will be performed in Step 11.4 below.)

SCA 11.1 Power up and adjust the variable frequency drive per VI 22515 12/4196 Supplement 15, Instruction Manua] D2-3233, Invertron VGI AC Drive 


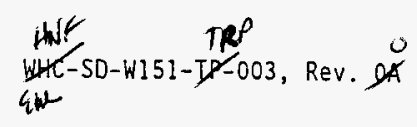

Controller Quick Start-Up Guide. Check all VFD parameters and record values in Appendix 0 .

Qw-11/4h 9611.2 Verify pump has been decoupled, bump motor, and verify direction as viewed from the top of the motor is clockwise.

ave $11 / 14$ h6 11.3 Check phase rotation.

$30124 / \% 11.4$. Perform motor tuning per vendor instructions (Instruction Manual D2-3233, page 3-3).

entand

11.5 With motor operating, back off the Vibraswitch setpoint screw one turn CCW and press reset button. If it does not reset, continue backing one turn and resetting until it does reset. Record the number of full CCN turns needed prior to being able to reset. After it resets, slowly turn screw CW until activation occurs.. Mark this position as "normal." If the "normal" setpoint screw position is less than $1.0 \mathrm{~g}$ (one full revolution), rotate the screw $0.5 \mathrm{~g} \mathrm{CCW}$. If "normal" is greater than $1.0 \mathrm{~g}$, refer to VI 22515 Supplement 32, book 3, tab 12, last page for final setpoint adjustment.

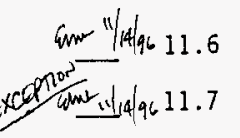

Check emergency stop function stops motor operation.

Check control console start and stop functions.

12. Check operation of main Pump 2 drive - observe for interference with instrumentation signals (e.g., harmonic interference). Note breaker $B 7$ in MCC-701 is closed, DS-701A is closed, and pump coupler is removed and locked out. (Note, any Vibraswitch lights turning on should be ignored as the final adjustment will be performed in Step 12.4 below.)

Power up and adjust the variable frequency drive per VI 22515 Supplement 15, Instruction Manual D2-3233, Invertron VGI AC Drive Controller Quick Start-Up Guide. Check all VFD parameters and record values in Appendix $E$.

Qw/ $15 / 126$ Verify pump has been decoupled, bump motor, and verify direction as viewed from the top of the motor is clockwise.

Qu/listir 12.3 Check phase rotation.

Jus akfor 12.4 Perform motor tuning per vendor instructions (Instruction Manual D2-3233, page 3-3).

12.5 With motor operating, back off the Vibraswitch setpoint screw one turn CCW and press reset button. If it does not reset, continue backing one turn and resetting until it does reset. Record the 


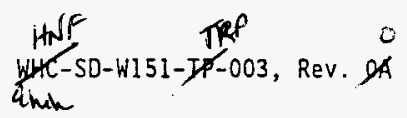

number of full CCW turns needed prior to being able to reset. After it resets, slowly turn screw CW until activation occurs. Mark this position as "normal." If the "normal" setpoint screw position is less than $1.0 \mathrm{~g}$ (one full revolution), rotate the screw $0.5 \mathrm{~g} \mathrm{CCW}$. If "normal" is greater than $1.0 \mathrm{~g}$, refer to VI 22515 supplement 32 , book 3 , tab 12 , last page for final setpoint adjustment.

Q

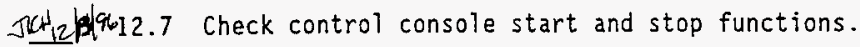




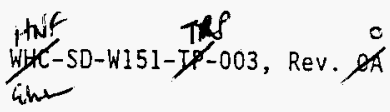

Appendix A - Checkout-Testing Exceptions

\begin{tabular}{|c|c|c|c|}
\hline \multicolumn{4}{|c|}{ CHECKOUT-TESTING EXCEPTION } \\
\hline $\begin{array}{l}\text { STEP } \\
\text { NO }\end{array}$ & DESCRIPTION OF PROBLEM & ACTION TAKEN & $\begin{array}{l}\text { RECHECK } \\
\text { COMPLETED }\end{array}$ \\
\hline 8.1 .1 & $\begin{array}{l}\text { WIRES ARE MIS-CABELHS UN EMERGENCY } \\
\text { SOP SWITCH }\end{array}$ & 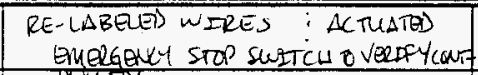 & $11 / 19 / 26$ \\
\hline 8.2 .1 & 11 & 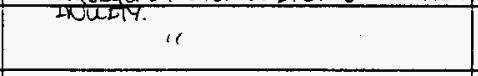 & $9 / 96$ \\
\hline 8.3 .1 & " & " & \\
\hline 8.4 .1 & $" 1$ & 14 & $9 \longdiv { 9 6 }$ \\
\hline 0.5 .2 & 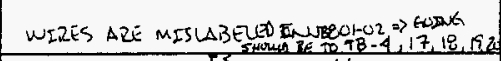 & & 2.196 \\
\hline 11.4 & 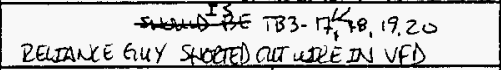 & REP (ALCE) WERE & 96 \\
\hline $\left.\begin{array}{l}12.7 \\
11.9\end{array}\right\}$ & 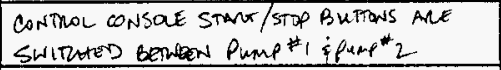 & 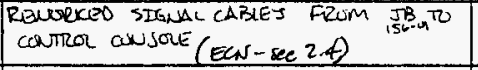 & $13 / 96$ \\
\hline $\begin{array}{l}8.5 .1 .2 \\
8.5 .1 .3\end{array}$ & 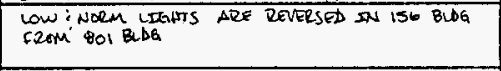 & 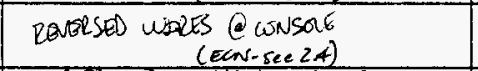 & \\
\hline 8.5 .1 .1 & $\begin{array}{l}\text { TUBANG IS REVERSED GUDNG DNTO ADFERENTAL } \\
\text { PRESSURE SWITLA, NU DOWR UIRE GOES }\end{array}$ & 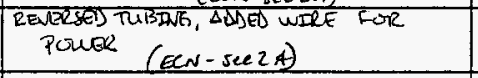 & $12 / 12 / 96$ \\
\hline & To suvitat & & \\
\hline 8.6 .1 & 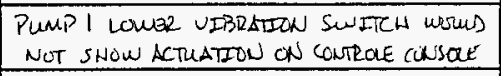 & $\begin{array}{l}\text { ELETRICDNLS INSTALLO JUMPER } \\
\text { THAT WAS MISSING }\end{array}$ & $12 / 13196$ \\
\hline 9.1 .5 & 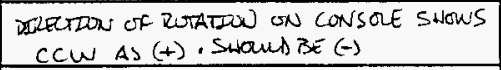 & CORIRETES METBR ON CONLOLG & $1 / 10$ \\
\hline 9.1 .9 & $\begin{array}{l}\text { OVRTRALR } \\
\text { SUICW'S WNN'T ACTUATE UNLESS }\end{array}$ & $\begin{array}{l}\text { ECN-WTRED THEM SO SERTES } \\
(\sec 24)\end{array}$ & $1 / 10 / 97$ \\
\hline 9.2 .10 & 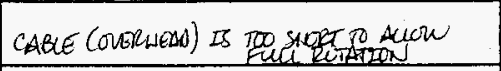 & $\begin{array}{l}\text { PUWUD SLACX CUT OF CABLE TO } \\
\text { DUCRGASE THE LENGTH ON TOP }\end{array}$ & $1 / 16 / 97$ \\
\hline 8.7 & $\begin{array}{l}\text { CABUE BETUEEN PUMP 1: ReMP 2JB'S IS } \\
\text { BAD. }\end{array}$ & RE-PUWED NEN WIRE & $1 / 7 / 97$ \\
\hline 8.7 & DIGITA TENSMMEER IN JBIOI-10 NOENWT & REPLALE DSEITAL TRANIMLTIER & \\
\hline
\end{tabular}




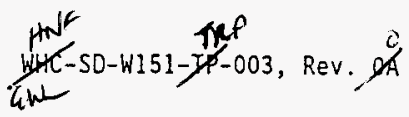

Appendix A - Checkout-Testing Exceptions

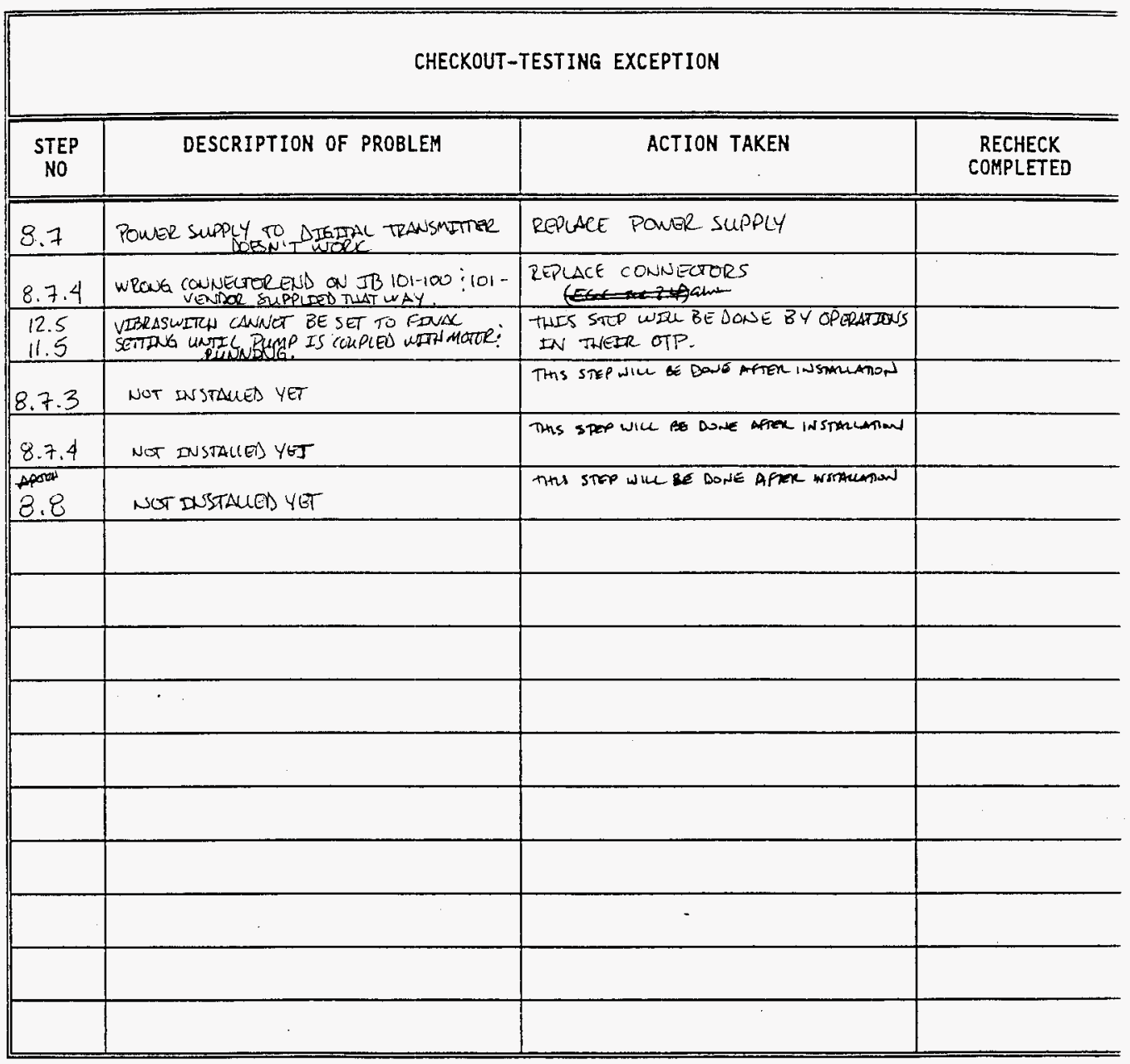




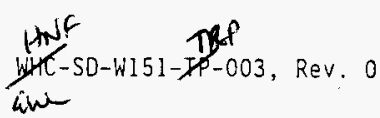

Appendix B - Pump 1 Rotation Drive Checklist

\begin{tabular}{|c|c|c|c|}
\hline \multicolumn{4}{|c|}{ CONTROL CONSOLE TO VFD-704A WIRING CHECK LIST } \\
\hline FROM & TO & VIA & $\checkmark$ \\
\hline DM2 Pg-2 & VFD $2 T B-110$ & TB4-12, $5156-6, M, 6-R E D$ & \\
\hline DM2 Pq-3 & VFD $2 T B-111$ & TB4-1IJJ156-6!L, 6-BLK & \\
\hline DM3 20 & VFD T2-20 & TB4-7,J156-6 G, 4-BLK & \\
\hline DM3 31 & VFD 31 & TB4-1, J156-6:A, 1-BLK & \\
\hline DM3 32 & VFD 32 & TB4-3,J156-6, C, 2-BLK & \\
\hline DM3 33 & VFD 33 & TB4-5, j156-6 E, 3-BLK & \\
\hline DM3 34 & VFD 34 & TB4-2\}J156-6 B, 1-RED & \\
\hline DM3 34 & VFD 34 & TB4-4 J156-6 D, 2-RED & \\
\hline DM3 34 & VFD 34 & TB4-6,J156-6 F, 3-RED & \\
\hline DM3 34 & VFD 21 & TE4-8,J156-6 H, 4-RED & \\
\hline DM3 34 & VFD 36 & TB4-10) J156-6, K, 5-RED & \\
\hline DM3 35 & VFD 35 & TB4-9,J156-6, J, 5-BLK & \\
\hline DM1 1 & VFD 1 & TB2-1,J156-5/A, 1-BLK & \\
\hline DM1 2 & VFD 2 & TB2- $2, J 156-5, B, 1-R E D$ & \\
\hline DM1 3 & VFD 3 & $T B 2-3, J 156-5, C, 2-8 L K$ & \\
\hline OM1 4 & VFD 4 & TB2-4,J156-5 D, 2-RED & \\
\hline OMl 12 & VFD 12 & TB2-7,J156-5 G, 4-BLK & \\
\hline DM1 13 & VFD 13 & TB2-8,J156-5/H, 4-RED & $\checkmark$ \\
\hline DM1 14 & VFD 14 & TB2-9,J156-5 J, 5-BLK & \\
\hline DMl 15 & VFD 15 & TB2-10 J156-5/K, 5-RED & $\checkmark$ \\
\hline OM1 16 & VFD $2 T B 6$ & TB2-11,JI56-5 L, 6-BLK & $\checkmark$ \\
\hline EMERG STOP 5 & VFD 11 & $T B 2-5, J 156-5, E, 2-B L K$ & \\
\hline EMERG STOP 6 & VFD 3CR WIRE 6 & TB2-6 $556-5 \mathrm{~F}$ & \\
\hline
\end{tabular}

REFERENCES \ENDOR DATA, H-2-78948 SHT 6, H-2-78991 SHT4, H-2-78983 SHT 1-4

PERFORMED BX Datereninen $11-5-96$ 
Table 8-4. Record of User's Parameter Selectlons/AdJustments.

\section{WARNING}

TABLE 8-4 IS TO BE USED AS A RECORD OF THE USER'S PARAMETER SELECTIONSIADJUSTMENTS. BEFORE MAKING ANY ADJUSTMENTS TO THE DRIVE, REFER TO THE APPROPRIATE FUNCTION OESCRIPTION IN CHAPTER 5. FAILURE TO OBSERVE THIS PRECAUTION COULD RESULT IN BODILY INJURY.

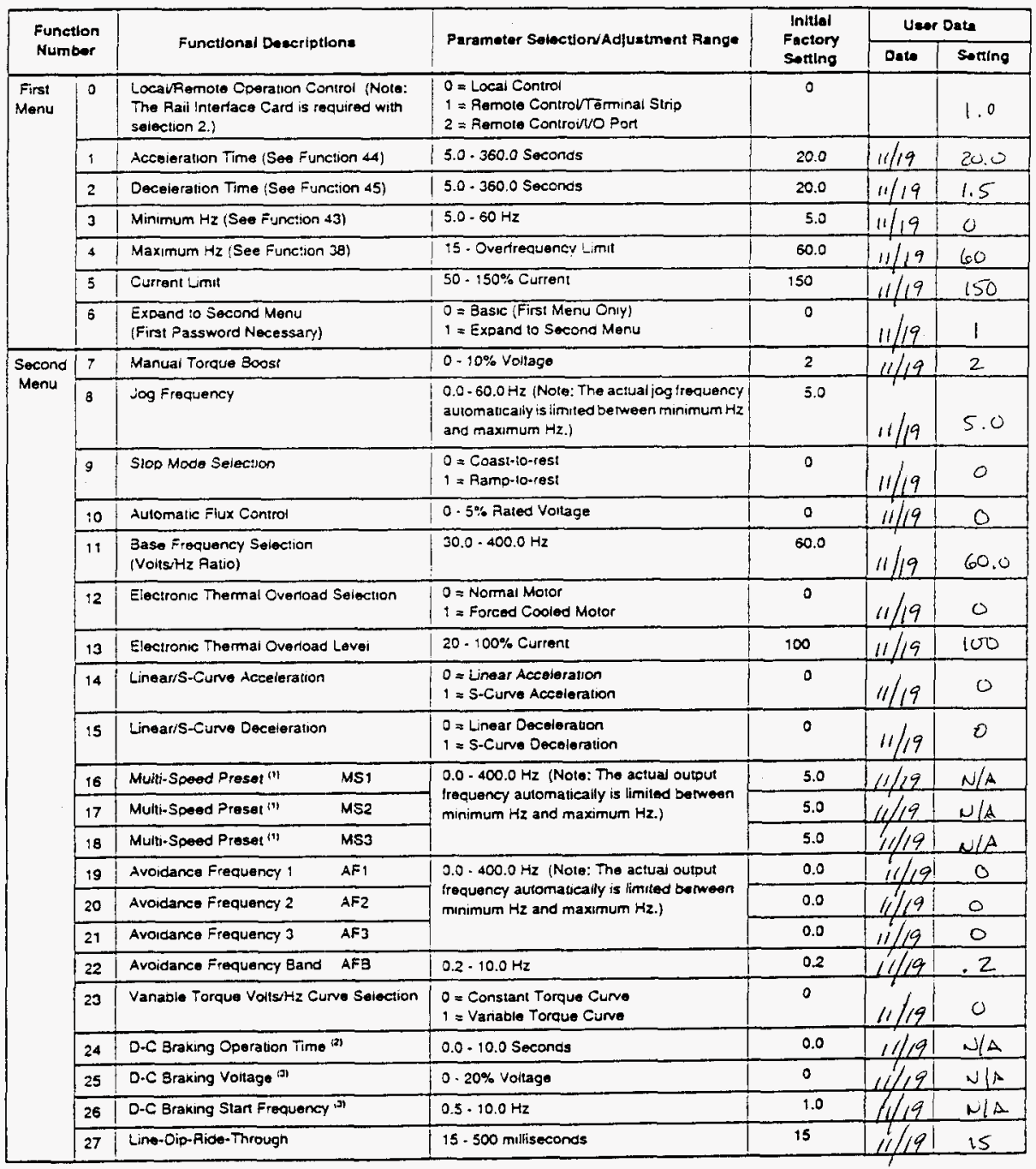


Appendix B - Rotation Drive Pump 1 Record Ctw

Table 8-4. Record of User's Parameter Selections/Ad]ustments (continued).

\begin{tabular}{|c|c|c|c|c|c|c|}
\hline \multirow{2}{*}{\multicolumn{2}{|c|}{$\begin{array}{l}\text { Function } \\
\text { Number }\end{array}$}} & \multirow{2}{*}{ Functional Descriptions } & \multirow{2}{*}{ Parameter SelectlonAdustment Range } & \multirow{2}{*}{$\begin{array}{l}\text { Initial } \\
\text { Factory } \\
\text { setting }\end{array}$} & \multicolumn{2}{|c|}{ Uear Data } \\
\hline & & & & & Dete & Sotting \\
\hline \multirow[t]{25}{*}{$\begin{array}{l}\text { Second } \\
\text { Menu } \\
\text { (cont.) }\end{array}$} & 28 & $\begin{array}{l}\text { Outout Relay-1 } \\
\text { (Form C Contact) } \\
\text { (Note: Requires the Remote Meler Inter. } \\
\text { (ace Card.) }\end{array}$ & \multirow{2}{*}{$\begin{array}{l}0=\text { Not Used } \\
1=\text { Zero Soeed Detect } \\
2 \text { = Resened } \\
3=\text { Outeut Contactor } \\
4 \text { a Frequency Lavel Detection } 1 \\
5=\text { Frequency Level Detection } 2 \\
6 \text { = Current Lovel Detection } \\
7 \text { = Reverse Rotation } \\
3=\text { D-C Braxing Operation } \\
9=\text { Reserved }\end{array}$} & 0 & & 0 \\
\hline & 29 & $\begin{array}{l}\text { Output Reiay } 2 \\
\text { (Form A Contact) } \\
\text { (Note: Pequires the Remote Meler Intep- } \\
\text { lace Card.) }\end{array}$ & & 0 & & 0 \\
\hline & 30 & Slip Compensation ${ }^{|4|}$ & $0.0-5.0 \mathrm{~Hz}$ & 0.0 & & 0.0 \\
\hline & 31 & $\begin{array}{l}\text { Inverse Reference is } \\
\text { (Second Pasaword Necessary) }\end{array}$ & $\begin{array}{l}0=\text { Normal } \\
1=\text { inverse }\end{array}$ & 0 & & $N \mid \Delta$ \\
\hline & 32 & $\begin{array}{l}\text { Function Lous Solection } \\
\text { (Second Password Nesessary) }\end{array}$ & $\begin{aligned} 0= & \text { IET at Function Loss } \\
1= & \text { Coast-10-rest without an IET output at } \\
& \text { Function Loss }\end{aligned}$ & 0 & & 0 \\
\hline & 33 & Frequency Levil Detection 1,81 & $0.5-405.0 \mathrm{~Hz}$ & 0.5 & & $\omega / A$ \\
\hline & 34 & Froquency Level Detection 2 in & $0.5 \cdot 405.0 \mathrm{~Hz}$ & 0.5 & & $N \mid \Delta$ \\
\hline & 35 & Current Level Detection (s) & $30-150 \%$ Current & 100 & & $N / A$ \\
\hline & 36 & Reverse Disabie & $\begin{array}{l}0=\text { Fonward Reverse Enaole } \\
1 \text { = Reverse Disable on Keypad }\end{array}$ & 0 & & 0 \\
\hline & 37 & $\begin{array}{l}\text { Automatic (Process Control) } \\
\text { Disade on Local Control }\end{array}$ & $\begin{array}{l}0=\text { AUTOMAN key Enable } \\
1=\text { AUTO Disabie on Keypad }\end{array}$ & 0 & & 0 \\
\hline & 38 & $\begin{array}{l}\text { Overtrequency Limit } \\
\text { (Second Password Necessary) }\end{array}$ & $50.0-405.0 \mathrm{~Hz}$ & 90.0 & & 90.0 \\
\hline & 39 & $\begin{array}{l}\text { D-C Otfset Enable } \\
\text { (Second Password Necessary) }\end{array}$ & $\begin{array}{l}0=\text { Cttset Disable } \\
1=\text { Ottset Enable }\end{array}$ & 0 & & 0 \\
\hline & 40 & $\begin{array}{l}\text { Auto-resel Enable } \\
\text { (Second Password Necessary) }\end{array}$ & $\begin{array}{l}0=\text { Auto-resel Disable } \\
1 \text { = Auto-resel Enade }\end{array}$ & 0 & & 0 \\
\hline & 41 & Auto-reset Time ("1) & 0 - 10 Times & 0 & & NIA \\
\hline & 42 & Auto-reset Interval Time i12) & $1-\infty$ Seconds & 1 & & $N / A$ \\
\hline & 43 & $\begin{array}{l}\text { Extended Minimum Hz Range } \\
\text { (Second Password Necessary) }\end{array}$ & $\begin{array}{l}0=\text { Disable }(5.60 \mathrm{~Hz}) \\
1=\text { Enable }(0.60 \mathrm{~Hz})\end{array}$ & 0 & & 1 \\
\hline & 4 & Extended Acceleration Time Rang* & $\begin{array}{l}0=5.0 \cdot 360.0 \text { Seconds } \\
1=0.1 \cdot 360.0 \text { Seconds }\end{array}$ & 0 & & 0 \\
\hline & 45 & Extended Decoleration Time Range & $\begin{array}{l}0=5.0 \cdot 360.0 \text { Seconds } \\
1=0.1 \cdot 360.0 \text { Seconds }\end{array}$ & 0 & & e 1 \\
\hline & 46 & RPM Monitor Dispiay Enable & $\begin{array}{l}0=\text { Disable } \\
1=\text { Enable }\end{array}$ & 0 & & 0 \\
\hline & 47 & $\begin{array}{l}\text { RPM Monitor Range Selection } \\
\text { (Second Password Necessary) }\end{array}$ & $\begin{array}{l}0=150-9999 \text { RPM } \\
1=0.9999 \mathrm{APM}\end{array}$ & 0 & & $N / 4$ \\
\hline & 48 & $\begin{array}{l}\text { RPM Monitor Base Froquency Selection } \\
\text { (See Function 47) }\end{array}$ & 150.9999 RPM & 1750 & & $N / A$ \\
\hline & 49 & $\begin{array}{l}\text { Output Voltage Progulation Mode Selaction } \\
\text { (See Function 50) }\end{array}$ & $\begin{array}{l}0=\text { Proponional to Inout } \\
1=\text { Fixed to Max Voltage }\end{array}$ & 0 & & 0 \\
\hline & 50 & Maximum Voltago & $\begin{array}{l}190.0-230.0^{114} \\
380.0=460^{1141} \\
475-575^{119}\end{array}$ & $\begin{array}{l}230.0^{114} \\
460.0^{114} \\
575^{11 n}\end{array}$ & $11 / 19$ & $N / A$ \\
\hline & 51 & $\operatorname{Jog}$ Acceteration Value & $0.1-360.0$ Seconds & 20.0 & $11 / 19$ & 20.0 \\
\hline & 52 & Jog Decelerstion Value & $0.1-360.0$ Seconds & 20.0 & $11 / 19$ & 20.0 \\
\hline
\end{tabular}




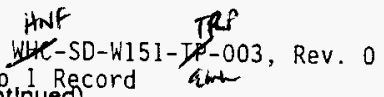

Appendix B - Rotation Drive Pump I Record Gut

Table 8-4. Record of User's Parameter Selectlons/Ad]ustments (continued).

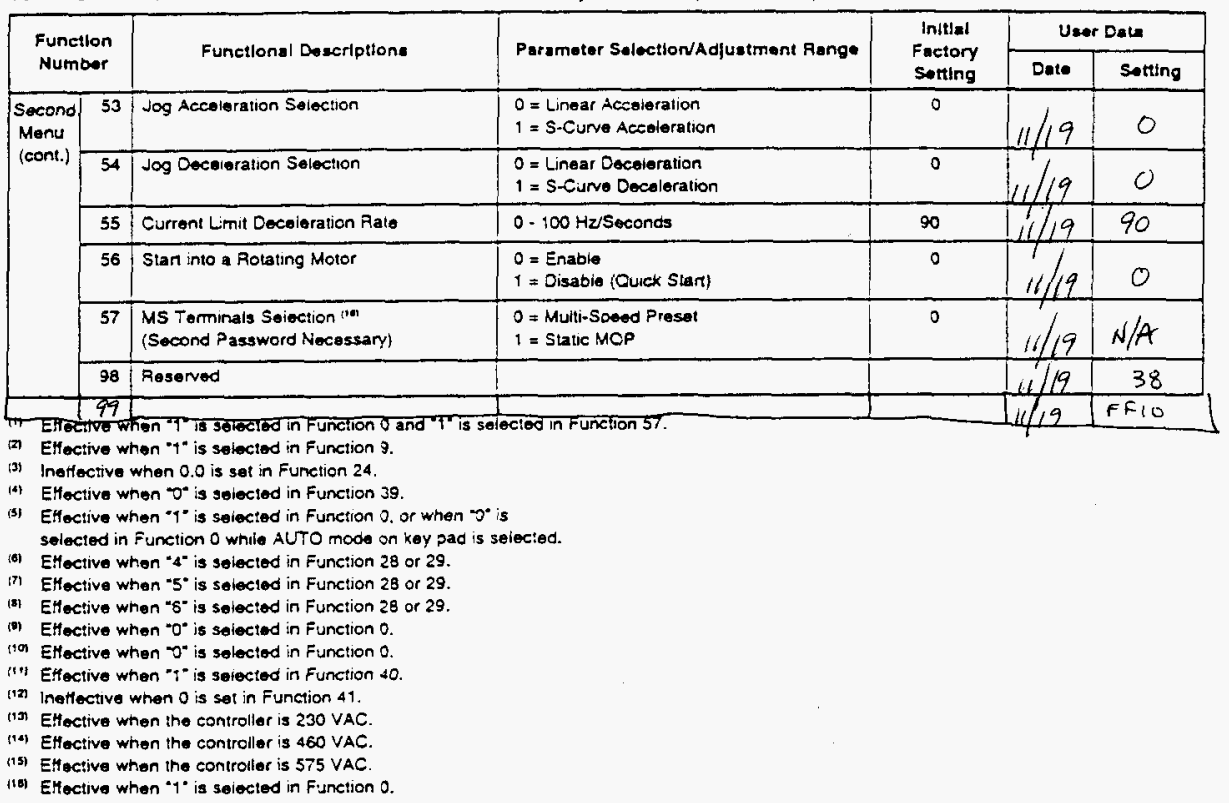


WITC-SD-W151- TP-003, Rev. 0

Appendix C - Pump 2 Rotation Drive Checklist

\begin{tabular}{|c|c|c|c|}
\hline \multicolumn{4}{|c|}{ CONTROL CONSOLE TO VFD-703A WIRING CHECK LIST } \\
\hline FROM & TO & VIA & $\sqrt{ }$ \\
\hline DM8 P9-2 & VFD $2 T B-110$ & TB9-12/J156-8/M, 6-RED & \\
\hline DM8 P9-3 & VFD $2 T B-111$ & TB9-11, J156-8! L, 6-BLK & \\
\hline DM9 20 & VFD T2-20 & TB9-7) $\Omega 56-8.6,4-B L K$ & \\
\hline DM9 31 & VFD 31 & TB9-1, J156-8 A, 1-BLK & \\
\hline DM9 32 & VFD 32 & TB9-3, $\sqrt{156-8} \mathrm{C}, 2-\mathrm{BLK}$ & \\
\hline DM9 33 & VFD 33 & TB9-5, $156-8 \mathrm{E}, 3-\mathrm{BLK}$ & \\
\hline DM9 34 & VFD 34 & TB9-2,J156-8,B, 1-RED & \\
\hline DM9 34 & VFD 34 & TB9-4,J156-8 D, 2-RED & \\
\hline DM9 34 & VFO 34 & TB9-6;J156-8 F, 3-RED & \\
\hline DM9 34 & VFD 21 & TB9-8, J150-8 H, 4-RED & \\
\hline DM9 34 & VFD 36 & T89-10,J156-8jK, 5-RED & \\
\hline DM9 35 & VFD 35 & TB9-9,J156-8,J, 5-BLK & \\
\hline DM7 I & VFD 1 & TB7-1 (J156-7) A, 1-BLK & \\
\hline DM7 2 & VFD 2 & TB7-2,J156-7 B, 1-RED & \\
\hline DM7 3 & VFD 3 & TB7-3 $3156-7, C, 2-B L K$ & \\
\hline DM7 4 & VFO 4 & TB7-4 JI56-7 D, 2-RED & \\
\hline DM7 12 & VFD 12 & TB7-7,J156-7 G, 4-BLK & \\
\hline DM7 13 & VFD 13 & TB7-8,J156-7, H, 4-RED & \\
\hline DM7 14 & VFD 14 & TB7-9; J156-7) J, 5-BLK & \\
\hline DM7 15 & VFD 15 & TB7-10, J156-7) K, 5-RED & \\
\hline DM7 16 & VFD 2 TB5 & TB7-11, J156-7 L, 6-BLK & \\
\hline EMERG STOP 7 & VFD 11 & $T B 7-5,156-7 \quad E, 2-B L K$ & \\
\hline EMERG STOP 8 & VFD 3CR WIRE 6 & TB7-6, $(156-7) F$ & $\checkmark$ \\
\hline
\end{tabular}

$\omega l a c$. $\omega / Q \infty \times C$

REFERENCES LUENDOR DATA, 78948 SHT 6, 78991 SHT3, 78983 SHT 1-4

PERFORMED BY De DATE(S) $11-5-96$ 


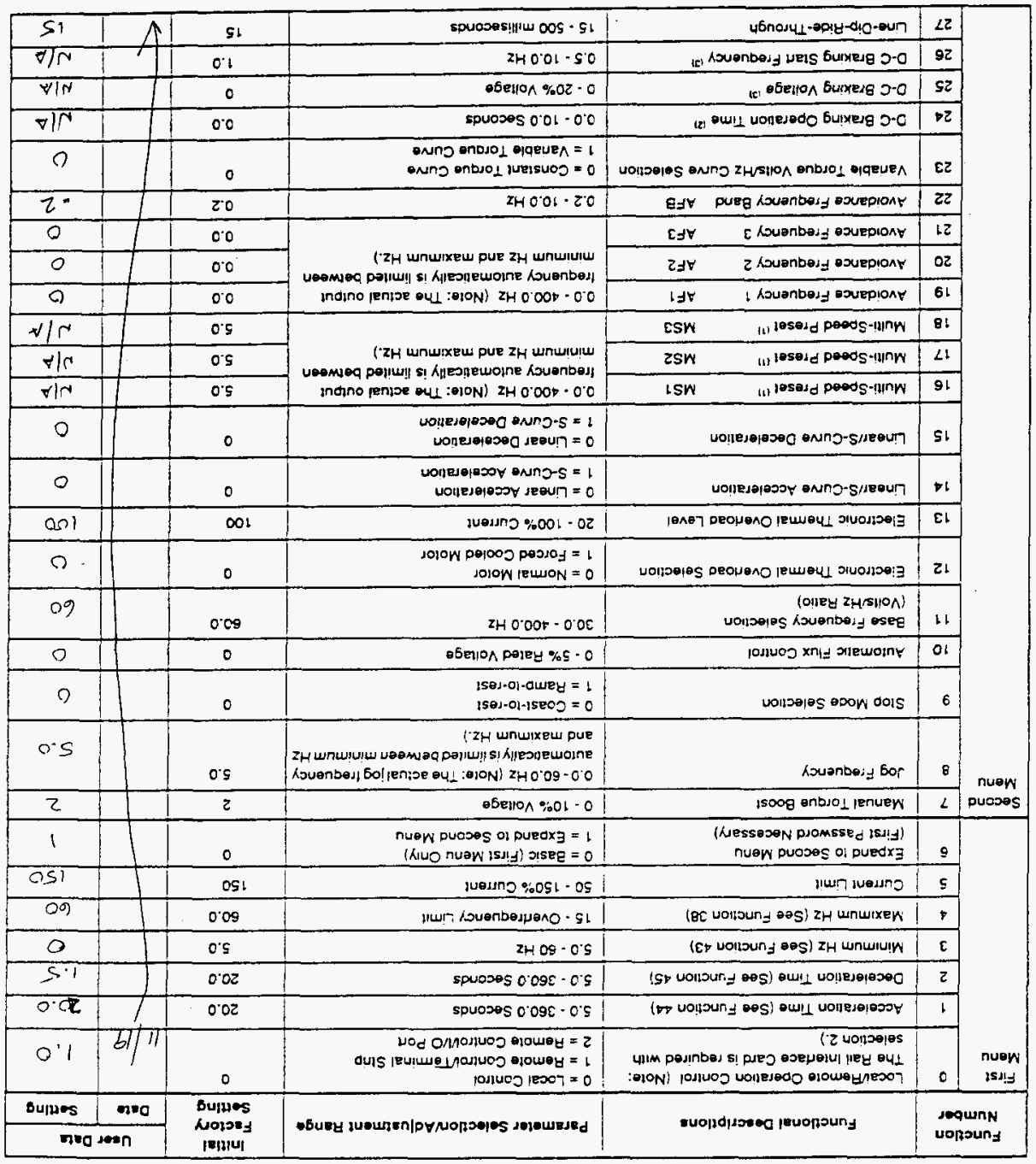




\section{$H+N$ \\ Wh-SD-W151-Te Th \\ Appendix C - Rotation Crive Pump 2 Record}

Table 8-4. Record of User's Parameter Selections/Adjustments (contInued).

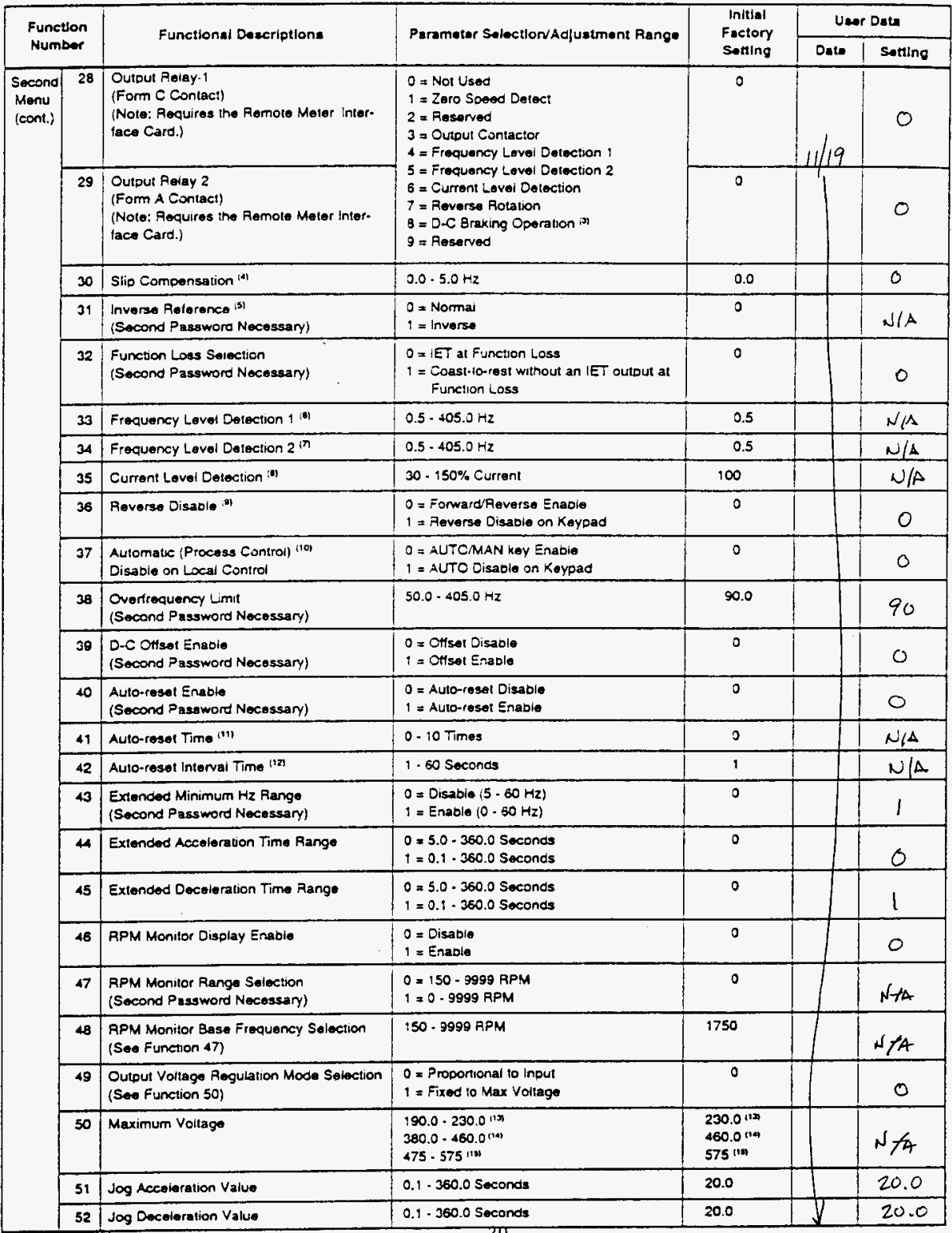


Appendix C - Rotation Drive Pump Wh 2 -SD-W151- XP-003, Rev. 0 Table 8-4. Record of User's Parameter Selections/Ad]ustments (continued).

\begin{tabular}{|c|c|c|c|c|c|c|}
\hline \multirow{2}{*}{\multicolumn{2}{|c|}{$\begin{array}{l}\text { Function } \\
\text { Number }\end{array}$}} & \multirow{3}{*}{ Functional Dexcriptions } & \multirow{3}{*}{$\begin{array}{l}\text { Parsmeter Seloction/Adfuztment Range } \\
\begin{array}{l}0=\text { Linear Acceieration } \\
1=S \text {-Curve Accoloration }\end{array}\end{array}$} & \multirow{3}{*}{$\begin{array}{c}\begin{array}{c}\text { Initial } \\
\text { Factory } \\
\text { Sotting }\end{array} \\
0\end{array}$} & \multicolumn{2}{|c|}{ Utor Dale } \\
\hline & & & & & \multirow{2}{*}{ Date } & \multirow{2}{*}{$\frac{\text { Sotting }}{0}$} \\
\hline Socond & 53 & & & & & \\
\hline & 54 & Jog Deceieration Selection & $\begin{array}{l}0=\text { Linear Deceiteration } \\
1=\text { S-Curve Decateration }\end{array}$ & 0 & & $\mathcal{O}$ \\
\hline & 55 & Current Limit Decoleration Rate & $0.100 \mathrm{~Hz} /$ Seconds & 90 & & 90.0 \\
\hline & 56 & Start into a Rotating Motor & $\begin{array}{l}0=\text { Enable } \\
1=\text { Disable (Quick Start) }\end{array}$ & 0 & & 0 \\
\hline & 57 & $\begin{array}{l}\text { MS Teminals Selection (1" } \\
\text { (Second Password Necessary) }\end{array}$ & $\begin{array}{l}0=\text { Muiti-Speed Preset } \\
1=\text { Static MOP }\end{array}$ & 0 & & $N / A$ \\
\hline & 98 & Reserved & & & & 38 \\
\hline & 99 & $+2=1$ & 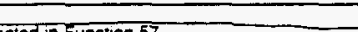 & & $\checkmark$ & FFIO \\
\hline
\end{tabular}

(2) Effoctive when " 1 " is selected in Function 9.

(3) Inerfective when 0.0 is sel in Function 24.

(4) Elfoctive when $0^{\circ}$ is serecied in Function 39.

(5) Elfective when " 1 " is selected in Function 0 , or when $O^{*}$ is solected in Function 0 while AUTO mode on key pad is selected.

(a) Effective when $44^{-1}$ is selected in Function 28 or 29.

in Eflective when " 5 " is selected in Function 28 or 29.

(8) Elfective when ${ }^{-6}-1$ is selectod in Function 28 or 29.

(2) Elfoctive when - $0^{*}$ is selocted in Function 0 .

(19) Elfective when ${ }^{\circ}$ is solectod in Function 0 .

(11) Elfoctive when " 1 " is seiected in Function 40.

ist Ineffective when $O$ is sel in Function 41 .

(1) Elfoctive when the controller is $230 \mathrm{VAC}$

(14) EMective when the controller is 460 VAC

(15) Effective when the controller is 575 VAC

(16) Elfective when " 1 " is selected in Function 0. 
Appendix 0 - Pump 1 Main Drive Checklist

\begin{tabular}{|c|c|c|c|}
\hline \multicolumn{4}{|c|}{ CONTROL CONSOLE TO VFD-702A WIRING CHECK LIST } \\
\hline FROM & IO & VIA & 1 \\
\hline DH10 P9-2 & $\begin{array}{l}\text { VFD TB } \times 9-552 \\
\text { (AMPS) }\end{array}$ & $\begin{array}{l}\text { IB7-17, RED, J/P156-9B, P/J156-14B, JBi56-01 TBZ-17, } \\
\text { RED }\end{array}$ & \\
\hline DH10 P9-3 & $\begin{array}{l}\text { VFD TB } \times 9-550 \\
\text { (COM) }\end{array}$ & $\begin{array}{c}\text { JB7-16, BLK, J/P156-9A, P/J156-14A, JB156-01 TB2-16, } \\
\text { BLK }\end{array}$ & \\
\hline OM11 PQ-2 & $\begin{array}{l}\text { VFD TB X9-551 } \\
\text { (VOLTS) }\end{array}$ & $\begin{array}{l}T B 7-20, R E D, J / P 156-9 E, P / J 156-14 E, J B 156-01 ~ T B 2-20, \\
\text { RED }\end{array}$ & \\
\hline 0M11 PQ-3 & $\begin{array}{l}\text { VFD TB } \times 9-550 \\
(\mathrm{CON})\end{array}$ & TB7-19, BLK, J/P156-90, P/J156-140, JB156-01 TB2-19, & \\
\hline DN12 PQ-2 & $\begin{array}{l}\text { VFD TB } \times 9-553 \\
\text { (FREO) }\end{array}$ & TB7-23, RED, J/P156-9H, P/J156-14H, JB156-01 TB2-23, & \\
\hline DM12 PQ-3 & $\begin{array}{l}\text { VFO TB } \times 9-550 \\
\text { (СCM) }\end{array}$ & TB7-22, BLK, J/P156-9G, P/J156-14G，J8156-01 TB2-22, & \\
\hline $\begin{array}{c}\text { METER WIRING SHIELDS NC } \\
\text { AT METERS }\end{array}$ & $\begin{array}{l}\text { VFD TB X9-157 } \\
\text { (SHIELDS) }\end{array}$ & $\begin{array}{r}\text { TB7-18, } 21,24 ; \text { J/P156-9C, F, J; P/J156-14C, F, J; } \\
\text { JB156-01 TB2-18,21, } 24 ; \text { PRAIN WIRES }\end{array}$ & \\
\hline $\begin{array}{c}\text { DSG }-1 \\
\text { (PUMP FAULT) }\end{array}$ & VFD IB $\times 7 \cdot F 1$ & $\begin{array}{c}\text { TB10-1, } 1 \text { BLK, J/P156-4A, P/J156-13A, JB156-01 TB2-1, } \\
\text { BLK }\end{array}$ & \\
\hline $054-2$ & VFD PACS $\times 8.20$ & TB10-2, I RED, J/P156-4B, P/J156-13B, JB156-01 IB2-2, & \\
\hline $\begin{array}{l}\text { DS3-1 } \\
\text { (PUNP RUNMING) }\end{array}$ & VFD PACS $\times 8-28$ & $\begin{array}{c}\text { TB10-3, } 2 \text { BLK, J/P156-4C, P/J156-13C, JB156-0I TB2-3, } \\
2 \text { BLX }\end{array}$ & \\
\hline DS3-2 & VFO PACS $\times 8-20$ & $\begin{array}{c}\text { TB10-4, 2 RED, J/P156-4D，P/J156-13D，JB156-01 TB2-4, } \\
2 \text { RED }\end{array}$ & \\
\hline DS3-1, DS4-1 & $\begin{array}{l}\text { HT-156-01 } \\
302,803\end{array}$ & (RS-485 INTERFACE) & \\
\hline $\begin{array}{l}\text { WT }-156-01 \\
\text { DATA, DATA* }\end{array}$ & $\begin{array}{l}\text { PC- } 1 \text { RS-485 PORT } \\
T X / R X+, T X / R X-\end{array}$ & TB3-2, TB3-1 & \\
\hline $\begin{array}{c}\$ 4-1 \\
(S T O P) \\
\end{array}$ & VFD PACS $\times 8-23$ & 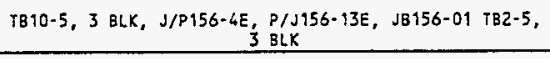 & \\
\hline$\$ 4-2$ & VFD PACS $\times 8-26$ & $\begin{array}{c}\text { TB10-6, } 3 \text { RED, J/P156-4F, P/J156-13F, JB156-0 TB2-6, } \\
3 \text { RED } \\
\end{array}$ & \\
\hline $\begin{array}{c}53-1 \\
\text { (START) }\end{array}$ & $\begin{array}{l}\text { VFD PACS } \times 8-29 \text { (JUMPER } \\
\text { TO PACS } \times 8-25 \text { ) }\end{array}$ & TB10-7, 4 BLK, J/P156-4G, P/J156-13G, JB156-01 TB2-7, & \\
\hline $53-2$ & VFD PACS $\times 8-23$ & 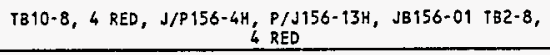 & \\
\hline $\begin{array}{l}\text { ES } 1-3 \\
\text { (EMERG. STOP) }\end{array}$ & VFD AUX TERM STRIP - 904 & TB10-9, 5 BLK, J/P156-4J, P/J156-J, JB156-01 TE2-9, 5 & \\
\hline ES1-4 & VFD AUX TERM STRIP - 22 & $\begin{array}{c}\text { tB10-10, 5-RED, J/P156-4K, P/J156-13K, JB156-01 } \\
\text { TB2-10,5 RED }\end{array}$ & \\
\hline SPARE & SPARE & $\begin{array}{c}\text { TB10-11, } 6 \text { BLK, J/P156-4L, P/J156-13L, JB156-01 } \\
\text { TB2-11, } 6 \text { BLK }\end{array}$ & \\
\hline SPARE & SPARE & $\begin{array}{c}1310-12, \text { \& RED, J/PiS6-4N; } \\
\text { T82-12, } 6 \text { RED }\end{array}$ & \\
\hline $\begin{array}{l}\text { SHIELD FOR } \\
\text { RED, BLK-1-6 }\end{array}$ & SHIELD & $\begin{array}{c}\text { TB10-13, DRAIN, J/P156-4N, P/J156-13N, JB156-01 } \\
\text { TB2-13, DRAIN }\end{array}$ & \\
\hline
\end{tabular}

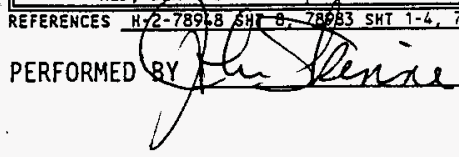

DATE(S) $11 / 5 / 96$ 


\section{WWH \\ Appendix D - Pump I Main Drive Record \\ Drive Record atmo}

Table 2-1. VGI Motor and Line Voltage Data and Volts/Hz Paramete: Quick Setup List.

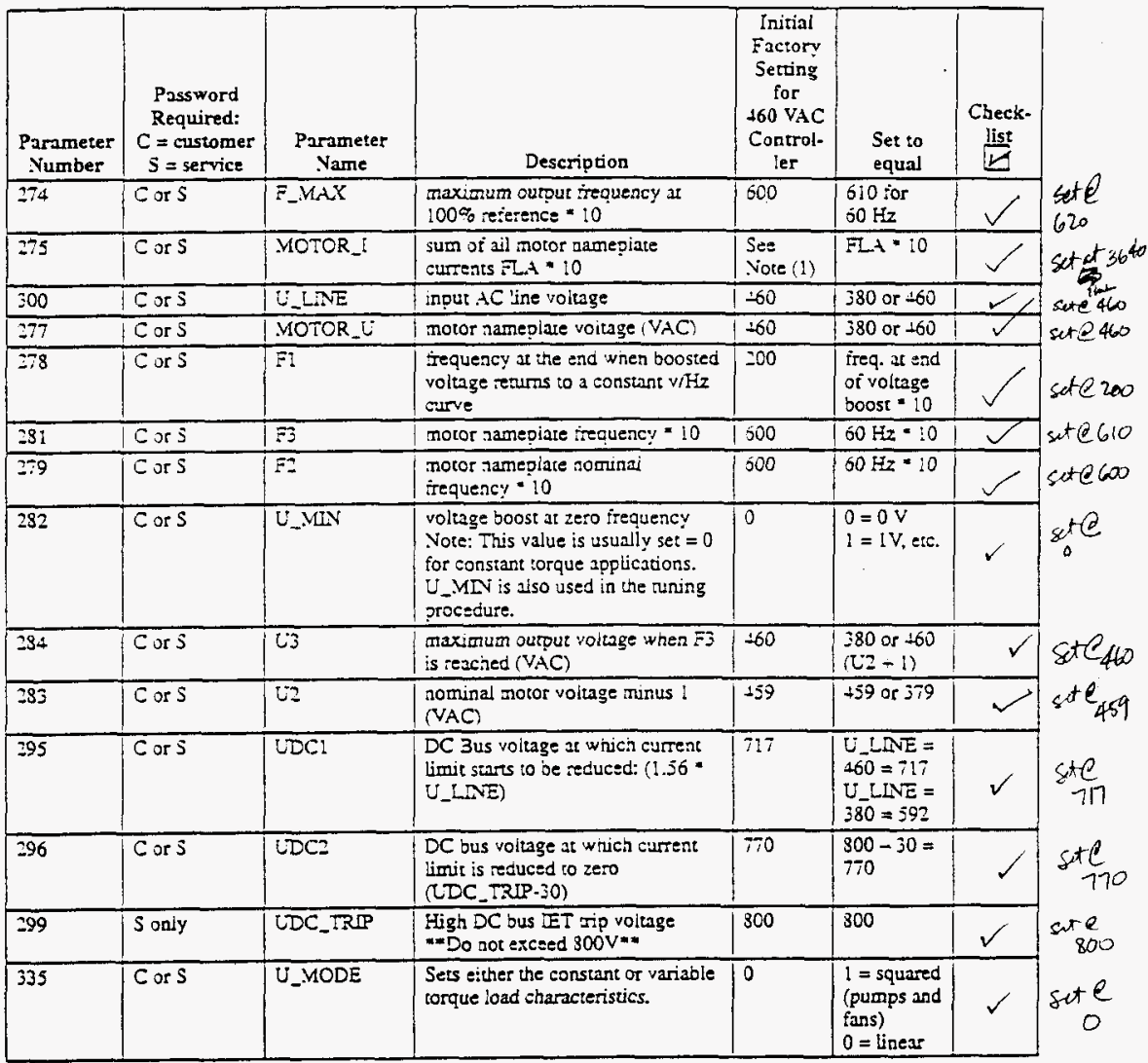

(1) 1800 for $150 \mathrm{HP}$ raring

2400 for $200 \mathrm{HP}$ raring

3000 for $30 \mathrm{HP}$ raring

3600 for 300 HP noing

4200 for $350 \mathrm{HP}$ rating

4800 for 400 HP raing

d. Recheck that all parameters in Table 2-1 have been eatered correctly. If you need all parameters values permanently stored, check that each parameter shows an " $=$ " sign next to the parameter value. If is does aot - (a slash mark " $P$ " is displayed instead), re-enter the value and press the FI key.

$$
\begin{aligned}
& \text { (2) Set pointer par ameter } 499 \text { to a value } 0 / 512 \text { to prevent reverse rotation selection } 11 / 15 / 96 \\
& \text { or keypel. ane } u /(5 / 96 \\
& \text { (3) final adjustimest to register } 368 \text { to a volve of } \frac{2284}{2458} \text { th set this }
\end{aligned}
$$




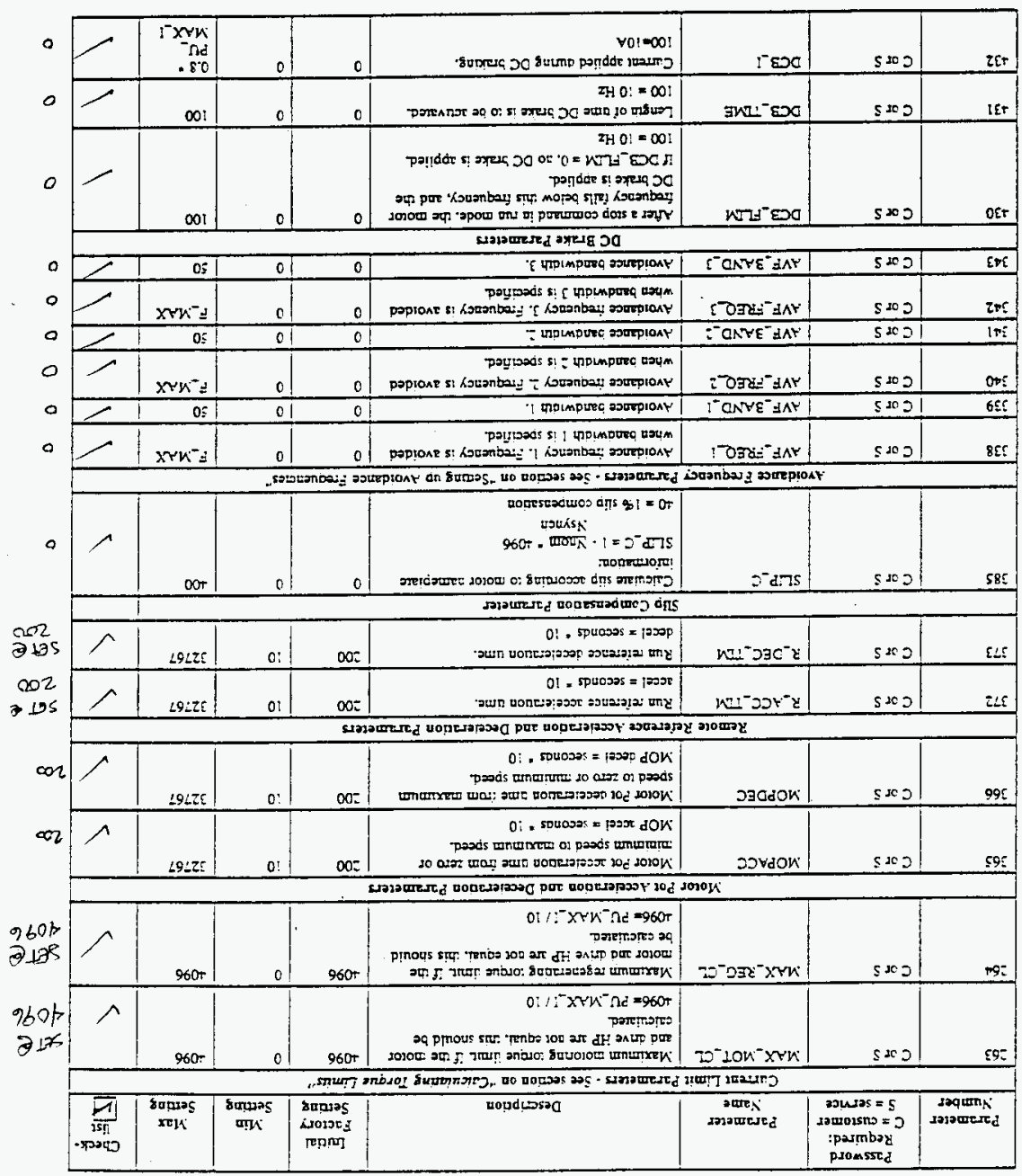

-2.

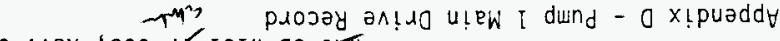

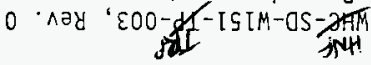


Table 3-1. VGI Ap̣olicanion Speciñc Parameter Settings. (Concinued)

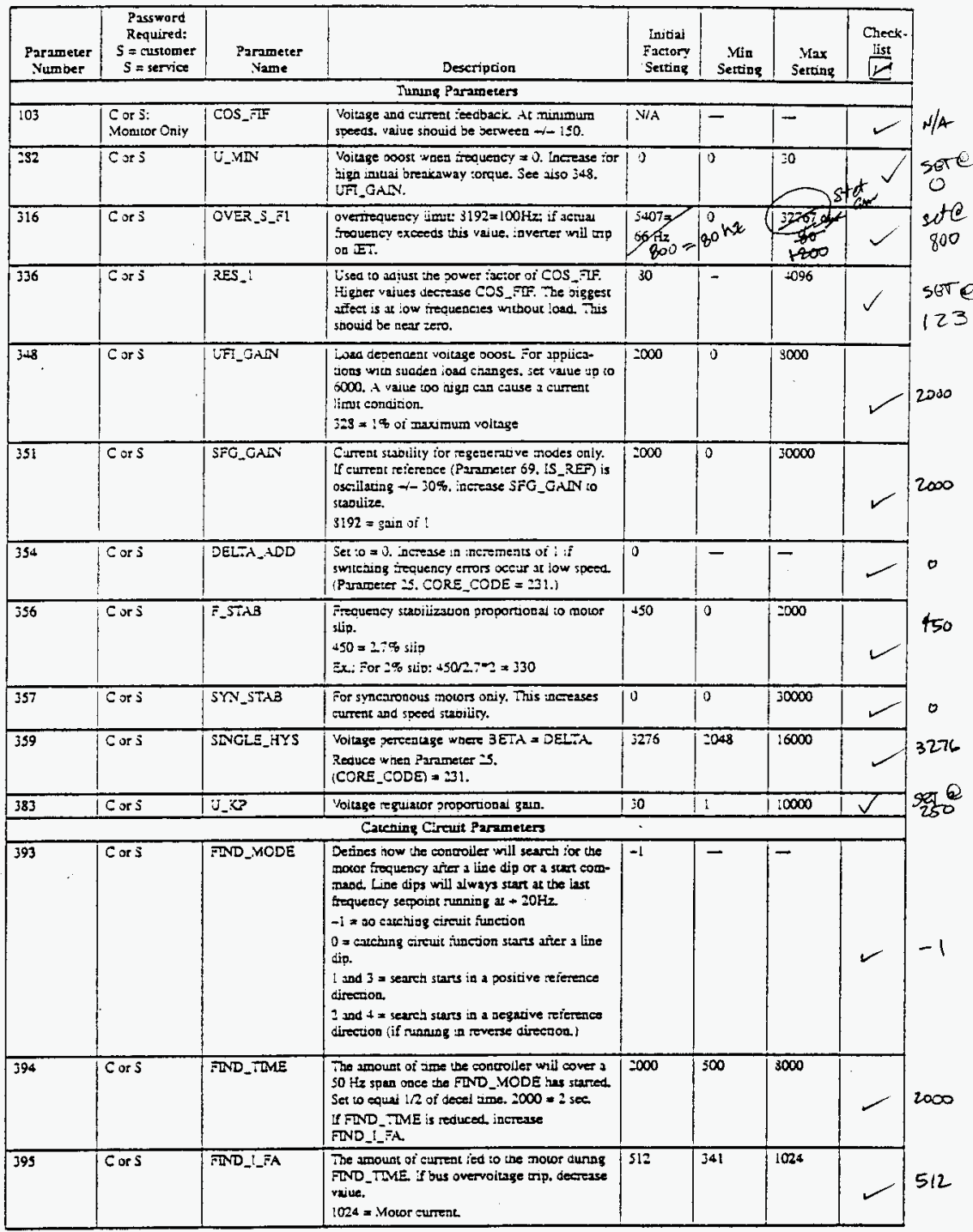




\section{Appendix D - Pump 1 Main Drive Record /}

Table 3-1. VGi Application Sp̣eciric Paramerer Sertings. (Continued)

\begin{tabular}{|c|c|c|c|c|c|c|c|}
\hline $\begin{array}{l}\text { Parameter } \\
\text { Vumper }\end{array}$ & $\begin{array}{l}\text { Pessword } \\
\text { Required: } \\
C=\text { customer } \\
\text { S = service }\end{array}$ & $\begin{array}{c}\text { Parameter } \\
\text { Name }\end{array}$ & Description & $\begin{array}{l}\text { Initial } \\
\text { Eactory } \\
\text { Setting }\end{array}$ & $\begin{array}{c}\text { Ytia } \\
\text { Setting }\end{array}$ & $\begin{array}{c}\text { Max } \\
\text { Serting }\end{array}$ & Cocek. \\
\hline 397 & Cors & $A F=A C C_{-} \cdot \bar{M}$ & 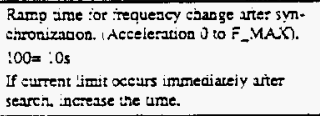 & 100 & $: 0$ & 10000 & \\
\hline \multicolumn{8}{|c|}{ Ramp or Coast Stop Parameter } \\
\hline+25 & Cors & RAMPSTP_EN & $\begin{array}{l}\text { Coast jtop (inmediate) or anp to rest stop. } \\
0=\text { coast stoo } \\
1=\text { ram to rest }\end{array}$ & 1 & 90 & 00 & \\
\hline
\end{tabular}

1. Calculating Torque Limits. If the drive and the notor are not matched in FP rating, or if there are multiple motors, the torque limits must be calculated.

Example Calculation:

VGI controiler is ated at $20 \mathrm{HP}$.

Motor FLA (from amepiace) $=14$ A.

Parameter 305 (PU_.MAX_I) $=400$. (The maximum output or the controller is 40 amps.)

Motor overcursear limit $=150 \%$, or $14 \times 1.5=21$ amps

In this case, the controller's output current ( 10 amps) can be greater than the motor's rated current ( 21 amps).

The maximum torcue limits for parmeters 263 (MAX_MOT_CL), and 264 (MAX_REG_CL) is 2096 . You need so calculate a new aumber for the torque limits for Parameters $26 \overline{3}$ (MLAX_MOT_CL), and 264 (MLAX_REG_CL) using the following equation:

$\frac{4096}{\text { Wax. Controller Output (Amps) }}=$
Example: $\quad \frac{4096}{40}=\frac{x}{21}$

$\frac{X}{\text { Motor full load amps }}$

$x=2150$

Enter 2150 into parameters 263 and 264 to limit the oupput of the controller to 21 amps.

For multiple motors: Use the sum of the full load motor amps of the motors.

\section{Tuning}

Note: It is recommended that this uning procedure be performed with an unloaded motor. If the motor cannot be unioaded. Parameter 282 (U_MIN) might have to be incrased significandly to boost the starting torciue.

Note: The motor will have to be warm to perform this aning procedure correcty. It could ake 1-2 hours to warm the motor field properly. Periorn Siep a berore concinuing with the rest of the runing procedure.

a. Run the motor, unloaded at $10 \mathrm{~Hz}$. Psiorm the following steps:

1. Monitor the OUTP AVPS using the display mode on the keypad

***Note: You cannot change Parameter 282 (U_MDN) while the controller is unning. You have to stop the controller, change U_MI, and restart *** 
Appendix E - Pump 2 Main Drive Checklist

\begin{tabular}{|c|c|c|c|}
\hline \multicolumn{4}{|c|}{ CONTROL CONSOLE TO VFD-701A WIRING CHECK LIST } \\
\hline FROM & To & VIA & 1 \\
\hline DM4 P9-2 & $\begin{array}{l}\text { VFD TB } \times 9-552 \\
\text { (AMPS) }\end{array}$ & $\begin{array}{c}\text { TB2-i7, RED, J/P156-2B, P/J156-11B, JB-156-01 TB1-17, } \\
\text { RED }\end{array}$ & \\
\hline aMG $P 9-3$ & $\begin{array}{c}y \in 2 \text { TR } \times 0-550 \\
\text { (CСA) }\end{array}$ & 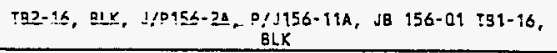 & \\
\hline DM5 PQ-2 & $\begin{array}{l}\text { VFD TB } \times 9.551 \\
\text { (VOLTS) }\end{array}$ & TB2-20, RED, J/P156-2E, P/J156-11E, JB156-01TB1-20, & \\
\hline 0M5 P9-3 & $\begin{array}{l}\text { VFD TB } \times 9-550 \\
(\mathrm{COM})\end{array}$ & TB2-19, 3LX, J/P156-2D, P/J156-110, JB156-09 TB1-19, & \\
\hline DMG PQ.2 & $\begin{array}{l}\text { VFD } \text { - B } \times 9-553 \\
\text { (FREO) }\end{array}$ & TB2-23，RED，J/P156-2H，P/J156-11H，JB156-01 TB1-23, & \\
\hline DKS PQ.3 & $\begin{array}{l}\text { VFD }+3 \times 9-550 \\
\text { (CON) }\end{array}$ & TB2-22, 8LK, J/P156-2G, P/J156-116, JB156-01 TB ${ }_{B L K}$ & \\
\hline $\begin{array}{l}\text { METER WIRING SHIELOS NC } \\
\text { AT METERS }\end{array}$ & $\begin{array}{l}\text { VFD IB } \times 9 \cdot 157 \\
\text { (SH:ELDS) }\end{array}$ & $\begin{array}{r}\text { TB2-18, 21, 24; J/P156-2C, F, J; P/J156-11C, F, J; } \\
\text { JB156-01 TB1-18,21，24; DRAIN WIRES }\end{array}$ & \\
\hline $\begin{array}{l}\text { DS2-1 } \\
\text { (PUMP FAULT) }\end{array}$ & VFD TB $\times 7-F 1$ & $\begin{array}{c}\text { IB3-17, } 1 \text { BLK, J/P156-3A, P/Ji56-12A, J8156-01 TB1-1, } \\
\text { i BLK }\end{array}$ & \\
\hline$D S 2-2$ & VFD PACS $\times 8-20$ & TB3-18， 1 RED，J/P156-3B，P/J156-12B，JB356-01 TB1-2, & \\
\hline $\begin{array}{c}\text { DS } 1-1 \\
\text { (PUMP RUNNING) }\end{array}$ & VFD PACS $\times 8-28$ & TB3-19, 2 BLK, J/P156-3C, ${ }_{2}^{P / J 156-12 C, J B 156-01 ~ T B 1-3, ~}$ & \\
\hline DS $1-2$ & VFD PACS $\times 8-20$ & T83-20, 2 RED, J/P156-30, P/J156-12D, JB156-01 TB1-4, & \\
\hline$D S 1-1,0 S 2-1$ & $\begin{array}{r}\text { WT-156-01 } \\
300,801 \\
\end{array}$ & (RS-485 INTERFACE) & \\
\hline $\begin{array}{l}\text { WT-156-01 } \\
\text { DATA, DATA" }\end{array}$ & $\begin{array}{l}\text { PC+1 RS }-485 \text { PORT } \\
\text { TX/RX+, TX/RX- } \\
\end{array}$ & $783-2, T B 3-1$ & \\
\hline $\begin{array}{l}S 2-1 \\
(S T O P)\end{array}$ & VFO PACS $\times 8-23$ & $\begin{array}{r}T B 3-21,3 \text { BLK, J/P156-3E, P/J156-12E，JB156-01 TB1-5, } \\
3 \text { BLK }\end{array}$ & \\
\hline $\mathrm{s} 2-2$ & VFD PACS $\times 8-24$ & 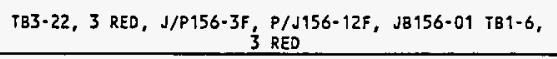 & \\
\hline $\begin{array}{c}\text { S1-1 } \\
\text { (START) }\end{array}$ & $\begin{array}{l}\text { VFO PACS } \times 8 \cdot 29 \text { (JUMPER } \\
\text { TO PACS } \times 8 \cdot 25 \text { ) } \\
\end{array}$ & TB3-23, 4 BLK, J/P156-3G, P/J156-12G, JB156-01 TB1-7, & \\
\hline$\$ 1-2$ & VFD PACS $\times 8-23$ & TB3-24, 4 RED, J/P156-3H, P/J156-12H, J8156-01 TB1-8, & \\
\hline $\begin{array}{c}\text { ES1-1 } \\
\text { (EMERG. STOP) }\end{array}$ & VFO AUX TERM STRIP - 904 & $\begin{array}{l}\text { TB5-1, } 5, B L K, J / P 156-3 J, P / J 156-12 J, J B 156-01 \text { TB1-9, } 5 \\
\text { BLK }\end{array}$ & \\
\hline$E 51-2$ & VFD ALXX TERM STRIP - 22 & TB5-2, 5 RED, J/P156-3K, P/J156-12K, JB156-01 TB1-10, & \\
\hline SPARE & SPARE & TB5-3, 6 BLK, J/P156-3L, ${ }_{6}^{P / J 156-12 L, J B 156-01 ~ T B 1-11, ~}$ & \\
\hline SPARE & SPARE & IE5-4, 6RED, J/P156-34, ${ }_{6}^{\text {PRED }}$ & \\
\hline $\begin{array}{l}\text { SHIELD FOR } \\
\text { RED, BLK } 1-6,0\end{array}$ & SHIELD & TB5-5, ORAIN, J/P156-3N, P/J156-12N, JB156-01 TB1-13, & \\
\hline
\end{tabular}




\section{HNE TH \\ Appendix E - Pump 2 Main Drive Record iqu}

Table 2-1. VGI Motor and Line Voltage Data and Volts/Hz Paramese: Quick Senp List.

\begin{tabular}{|c|c|c|c|c|c|c|c|}
\hline $\begin{array}{c}\text { Parameter } \\
\text { Yumber }\end{array}$ & $\begin{array}{c}\text { Password } \\
\text { Required: } \\
C=\text { ctrstomer } \\
\mathrm{S}=\text { service }\end{array}$ & $\begin{array}{c}\text { Parameter } \\
\text { Name } \\
\end{array}$ & Description & $\begin{array}{c}\text { initial } \\
\text { Factory } \\
\text { Serting } \\
\text { for } \\
+60 \mathrm{VAC} \\
\text { Control. } \\
\text { ler } \\
\end{array}$ & $\begin{array}{r}\text { Set to } \\
\text { equal } \\
\end{array}$ & $\begin{array}{c}\text { Check- } \\
\text { list } \\
\square\end{array}$ & \\
\hline 274 & $\operatorname{Cor} \mathrm{s}$ & F_MAX & $\begin{array}{l}\text { maximum output frequency as } \\
100 \% \text { referescs }=10\end{array}$ & 600 & $\begin{array}{l}610 \mathrm{ior} \\
60 \mathrm{izz} \\
\end{array}$ & & $\begin{array}{l}\text { SET } \\
620\end{array}$ \\
\hline 275 & Cor $S$ & MOTOR_L & $\begin{array}{l}\text { sum of sil motor aamepiate } \\
\text { currents Fly * } 10\end{array}$ & $\begin{array}{l}\text { Sete } \\
\text { Nore (1) }\end{array}$ & FIt $=10$ & $\checkmark$ & $S E T+3640$ \\
\hline 300 & Cors & U.LNE & input $A C$ line voltage & -60 & 380 or +60 & 1 & sote 400 \\
\hline 277 & Cors & MOTOR_L & motor namepiate voitage $\left(V_{A C}\right)$ & 160 & 380 or 160 & & $\operatorname{sen} 240^{\circ}$ \\
\hline 298 & $\operatorname{CorS}$ & $F I$ & $\begin{array}{l}\text { Trequency af the end when boosted } \\
\text { voltage rearns to a constant v/Hz } \\
\text { Jurve }\end{array}$ & 200 & $\begin{array}{l}\text { freq. ar end } \\
\text { of voltage } \\
\text { boost }=10\end{array}$ & 2 & set $e 20^{\circ}$ \\
\hline 291 & Cors & 83 & motor namepiare rirequency $* 10$ & 600 & $60 \mathrm{~Hz} * 10$ & 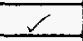 & SET C 610 \\
\hline 379 & Cors & $F 2$ & $\begin{array}{l}\text { notor nameglate nominal } \\
\text { requency }=10\end{array}$ & 600 & $60 \mathrm{~Hz} * 10$ & & sot e 000 \\
\hline 232 & Cors & U..VIN & $\begin{array}{l}\text { voltage boost at zero frequency } \\
\text { Noce: This value is usually set=0 } \\
\text { for constant torque apolications. } \\
\text { U_MNN is aiso used in the turing } \\
\text { procedure. }\end{array}$ & 0 & $\begin{array}{l}0=0 \mathrm{~V} \\
1=1 \mathrm{~V}, \text { etc. }\end{array}$ & & $\begin{array}{c}\text { sute } \\
0\end{array}$ \\
\hline 284 & Cor $S$ & 43 & $\begin{array}{l}\text { naximum ourpur voltage when F3 } \\
\text { is reached (VAC) }\end{array}$ & \pm 60 & $\begin{array}{l}380 \text { or }-60 \\
(\mathrm{~L} 2-1)\end{array}$ & & sज 2460 \\
\hline 293 & Cor $\$$ & $t_{2}$ & $\begin{array}{l}\text { nominal motor voltuge minus l } \\
\text { (VAC) }\end{array}$ & -159 & 459 or 379 & & $\begin{array}{l}56 \pi 2 \\
459\end{array}$ \\
\hline 395 & Cors & UDC! & 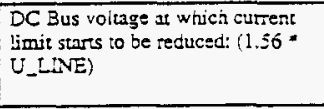 & 717 & $\begin{array}{l}U_{\text {LIVE }}= \\
460=717 \\
U_{2} \operatorname{LINE}= \\
380=592\end{array}$ & & $\begin{array}{l}58 \pi Q \\
717\end{array}$ \\
\hline 296 & Cors & UDC? & $\begin{array}{l}\text { DC bus voitsge at which current } \\
\text { limit is reduced to zero } \\
\text { (CDC_TRPP-jO) }\end{array}$ & 770 & $\begin{array}{l}800-50= \\
770\end{array}$ & & $\begin{array}{l}\text { sete } \\
770\end{array}$ \\
\hline 299 & S oniy & $\overline{U D C}$ _RIP & $\begin{array}{l}\text { High DC bus IET rip voltage } \\
\text { "Do aot exceed } 300 \mathrm{~V}^{* *}\end{array}$ & 300 & 300 & $\checkmark$ & sete \\
\hline 335 & Cors & U_MODE & $\begin{array}{l}\text { Sets either the constant or variable } \\
\text { torque load characteristics. }\end{array}$ & 0 & $\begin{array}{l}1=\text { squared } \\
\text { (pumps and } \\
\text { fans) } \\
0=\text { inear }\end{array}$ & & sore \\
\hline
\end{tabular}

(1) 1800 for $150 \mathrm{HP}$ raring

2400 for $200 \mathrm{HP}$ raning

3000 for $250 \mathrm{HP}$ rating

3600 for 300 HP rating

4200 for $350 \mathrm{HP}$ raing

4800 for $400 \mathrm{HP}$ rating

d. Recbeck that all parameters in Table 2-1 have been entered correcily. if you need all parameters values permanendy stored, check that each paraneter shows an " $=$ " sign next to the parameter value. If is does not - (a slash mark " $p$ " is displayed instead), re-enter the value and press the Fl key.

(2) Sot polnter parameter 499 to a vacve of 512 to prenant reverse rutatzon squctan 2-2 OR SOYPAD.

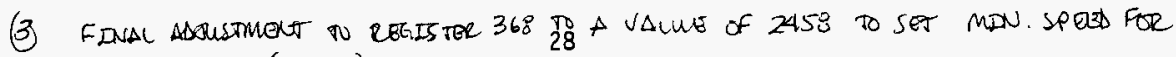
$712 \sin (36 \mathrm{~Hz})$ 
Appendix E - Pump 2 Main Drive Record

Table 3-1. VGI Applicacion Speciñc Parameter Setrings. (Continued)

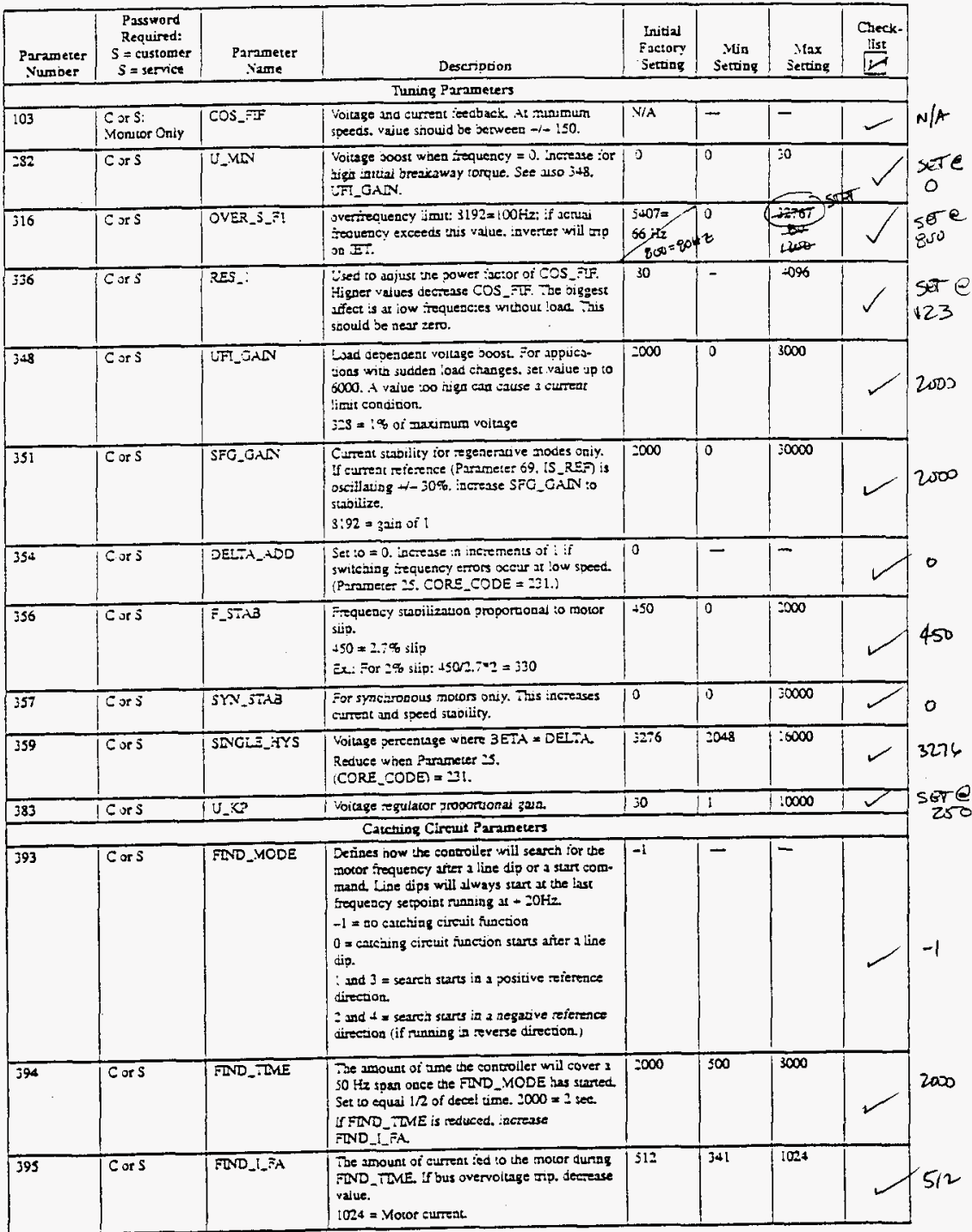




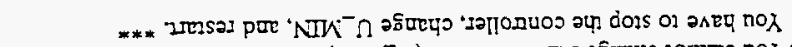

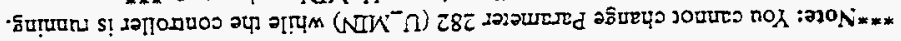

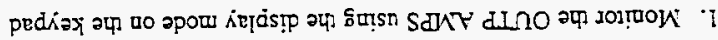

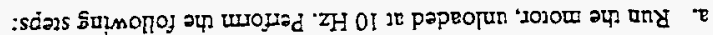

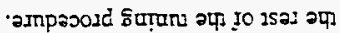

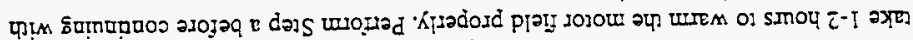

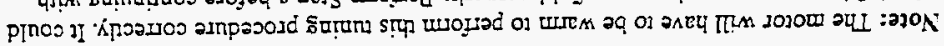

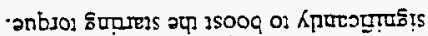

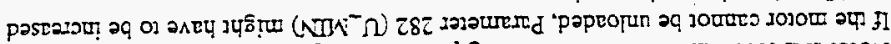

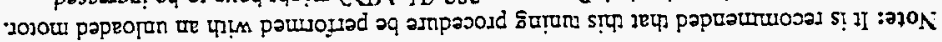

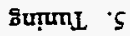

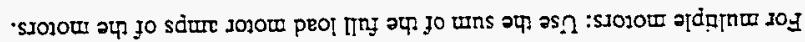

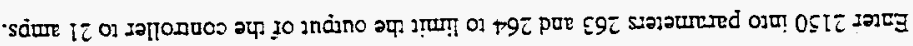

$$
\operatorname{OSIZ}=x
$$

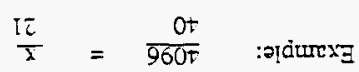

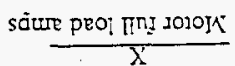

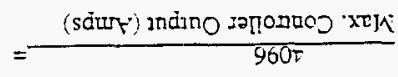

:uopenbe

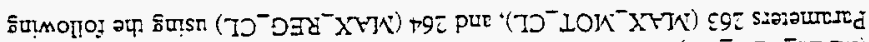

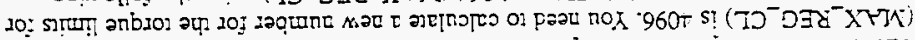
т9ட pसr '(

( (säure

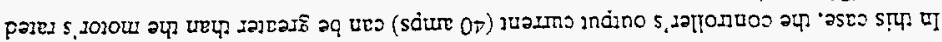

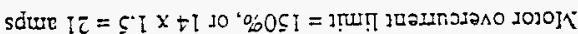

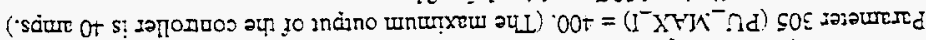

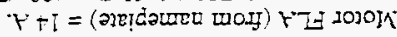

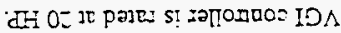

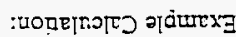

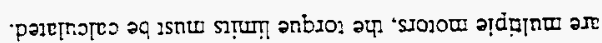

\begin{tabular}{|c|c|c|c|c|c|c|c|}
\hline & 00 & $\infty$ & $i$ & 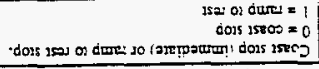 & 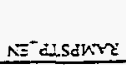 & $5+2$ & 525 \\
\hline \multicolumn{8}{|c|}{ 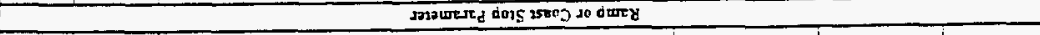 } \\
\hline & 0000: & 0 & $00:$ & 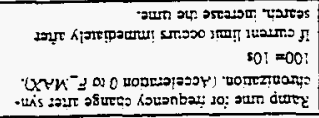 & 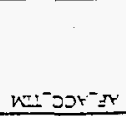 & 5100 & L6: \\
\hline 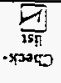 & $\begin{array}{c}\text { sugnas } \\
\text { xex }\end{array}$ & $\begin{array}{c}\text { Sugurs } \\
\text { tix }\end{array}$ & 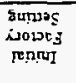 & uopt Lossa & 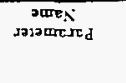 & 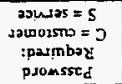 & $\begin{array}{l}\text { saquink } \\
\text { sasourited }\end{array}$ \\
\hline
\end{tabular}

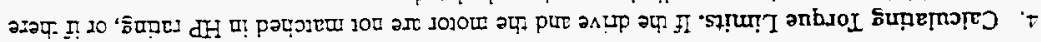

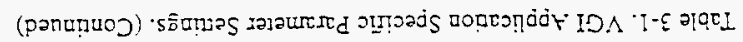

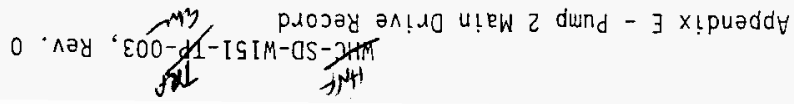


Appendix F - Pressure Switch Checklist

\begin{tabular}{|c|c|c|c|}
\hline \multicolumn{4}{|c|}{ COLUMN WATER PRESSURE SWITCHES WIRING CHECK LIST } \\
\hline FROM & TO & VIA & 1 \\
\hline PSL-TSO C, NE, WO, NONE & J8801-01 :8:-:;, -2, $+3,-4$ & 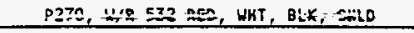 & \\
\hline PSL $=751 C, N C$, NO, NONE & J8801-01 TB1.5, $-6,-7,-8$ & $P 271, U / R 533$ RED, UHT, BLK, SHLD & \\
\hline PDIS $-750 \mathrm{C}$, NC, NO, NONE & $J B 801-01 Y 81-9,-10,-11,-12$ & P272 W/R 534 RED, WHT, BLK, SHLD & \\
\hline JB801-01 TB1-1, -2, -3, -4 & j8801-02 $181-1,-2,-3,-4$ & P269 U/R 531 1-BLK, HHT, RED, SHLD & \\
\hline JB801-01 TB1-5, -6, -7, -8 & J8801-02 $781+13,-14,-15,-16$ & P269 W/R 531 2-BLK, WHT, RED, SHLD & \\
\hline J8801-01 т81-9,-10,-11,-12 & $18801-02\left(T B \frac{2}{2}-1,-2,-3,-4\right.$ & P269 W/R 539 3-BLK, HHT, RED, SHLD & \\
\hline$J B 801-02$ TB1- $1,-2,-3,-4$ & J8101-100 $134-9,-10,-11,-12$ & P210 W/R 530 1-BLK, WHT, RED, SHLD & \\
\hline $3 B 801-02$ IB1-13,-14,-15,-16 & $J B 101-100$ I $86-13,-14,-15,-16$ & P210 W/R 530 4-BLK, HHT, RED, SHLD & \\
\hline JB801-02 TB2-1, $-2,-3,-4$ & $J 8101-100 T 84-17,-18,-19,-20$ & P210 H/R 530 7-BLK, HHT, RED, SHLD & \\
\hline JB109-100 TB4-9, $-10,-11,-12$ & J8156-01 T33-13,-14,-15,-16 & $P 206,261,301 \mathrm{~W} / R 504,10-B L K, W H T, R E D, S H L D$ & \\
\hline$J 8101-100 T B 4-13,-14,-15,-16$ & $J B 956-09 T 83-17,-18,-19,-20$ & P206,261,301 W/R $504: 11-8 L K, W H T, R E D$, SHLD & \\
\hline JB10 $-100 T 84-17,-18,-19,-20$ & $\pm 8156-01, T B 3-21,-22,-23,-24$ & P206,261,301 W/R $504 \quad 12-B L K, H H T, R E D$, SHLO & \\
\hline JB156-01 TB3-13, - 14, - 15, - 16 & $\begin{array}{l}\text { CONTROL CONSOLE } \\
785-13,16,15,16\end{array}$ & $\begin{array}{l}J / P 156-10 \text { A,B,C,D BLK, RED, CLR, SHLD, } \\
P / J 156-1 \text { A,B,C,D BLK, WHT, RED, SHLD }\end{array}$ & \\
\hline JB156-01 TB3-17, $-18,-19,-20$ & $\begin{array}{l}\text { CONTROL CONSOLE } \\
\text { TB5-17, } 18,99,20\end{array}$ & $\begin{array}{l}J / P 156-10 \text { E,F,G,H BLK, RED, CLR, SHLD, } \\
\text { P/J156-1 E,F,G.H BLX, WHT, RED, SHLD }\end{array}$ & \\
\hline JB156-01 TB3-21, $-22,-23,-26$ & $\begin{array}{c}\text { CONYROL CONSOLE } \\
\text { TB5-21, 22, 23, } 24\end{array}$ & $\begin{array}{r}J / P 156-10 \mathrm{~J}, K, L, M \text { BLK, RED, CLR, SHLD, } \\
\text { P/J156-1 J,K,L,M BLK, UHT, RED, SHLD }\end{array}$ & \\
\hline $\begin{array}{l}\text { CONTROL CONSOLE } \\
\text { TB5-13,-16,-15,-16 }\end{array}$ & PS-1+, DS7-1, DS8-1, NCNE & JMPR， BLK， RED，SHLD & \\
\hline $\begin{array}{l}\text { CONTROL CONSOLE } \\
\text { TB5-17,-18,-19,-20 }\end{array}$ & PS-1+, DS9-1, DS10-1, NONE & JMPR, BLK, RED, SHLD & \\
\hline $\begin{array}{l}\text { CONTROL CONSOLE } \\
\text { TB5-21, -22, }-23,-24\end{array}$ & PS-1+, 0S5-1, DS6-1, NONE & JMPR, BLK, RED, SHLD & \\
\hline DS7-1, DS8-1 & $W T-156-01-B 06,-B 07$ & (RS-485 INTERFACE) & \\
\hline $0 \$ 9-1,0510-1$ & $W T-156-01-808,-809$ & (RS -485 [NTERFACE) & \\
\hline DS5-1, $056-1$ & $4 T-156-01-804,-805$ & (RS-485 IHTERFACE) & \\
\hline WT=156-01 DATA, DATA* & PC- 1 RS485 PORT TX/RX+, IX/RX- & $T 83-2,-1$ & \\
\hline
\end{tabular}

REFERENCES H-2-78986 SHT 3, 78987 SHT 3,78948 SHT $4,10,78983$ SHT3, 4

PERFORMED BY N)/A DATE(S)

NOT USED. 8.5.2 SAYS TO USE ONLY IF PROBLOMS ARE ENCOUNTERED. 


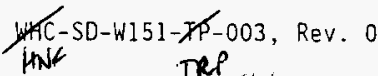

TRP ane

Appendix G - VibraSwitch Checklist

page 1 of 2

\begin{tabular}{|c|c|c|c|}
\hline \multicolumn{4}{|c|}{ PUMP \#1 ( P-702A) VIBRASWITCH WIRING CHECK LIST } \\
\hline FROM & To & VIA & $\checkmark$ \\
\hline $\begin{array}{l}\text { P1-VIB-1 UPPER } \\
\text { NO, COM, NC }\end{array}$ & $\begin{array}{l}\text { RTD JBOX TERMINALS } \\
14,15,16\end{array}$ & $\begin{array}{c}\text { P334 W/R } 561 \\
\text { BLK, CLR, RED } \\
\end{array}$ & \\
\hline $\begin{array}{l}\text { P1-VIB-2 LOWER } \\
\text { NO, COM, NC } \\
\end{array}$ & $\begin{array}{l}\text { RTD JBOX TERMINALS } \\
32,33,34 \\
\end{array}$ & $\begin{array}{c}\text { P331 W/R } 562 \\
\text { BLK, CLR, RED }\end{array}$ & \\
\hline $\begin{array}{l}\text { RTD JBOX TERMINALS } \\
14,15,16\end{array}$ & $\begin{array}{c}J B 101-101 \text { TB4- } \\
1,2,3,4\end{array}$ & $\begin{array}{l}\text { P333 P332 W/R } 560 \\
\text { 3-BLK, WHT, RED, SHLD }\end{array}$ & \\
\hline $\begin{array}{l}\text { RTD JBOX TERMINALS } \\
32,33,34 \\
\end{array}$ & $\begin{array}{c}\text { JB101-101 TB4- } \\
5,6,7,8 \\
\end{array}$ & $\begin{array}{l}\text { P333 P332 W/R } 560 \\
\text { 4-BLK, NHT, RED, SHLD }\end{array}$ & \\
\hline $\begin{array}{c}\text { JB101-101 TB4- } \\
1,2,3,4 \\
\end{array}$ & $\begin{array}{c}\text { JB101-100 TB4- } \\
1,2,3,4 \\
\end{array}$ & $\begin{array}{c}\text { P215 W/R } 528 \\
1-B L K, W H T, R E D, S H L D\end{array}$ & \\
\hline $\begin{array}{c}\text { JB101-101 TB4- } \\
5,6,7,8\end{array}$ & $\begin{array}{l}\text { JB101-100 TB4- } \\
5,6,7,8\end{array}$ & $\begin{array}{c}\text { P215 W/R } 528 \\
\text { 2-BLK, WHT, RED, SHLD }\end{array}$ & \\
\hline $\begin{array}{c}J 8101-100 \text { TB4- } \\
1,2,3,4 \\
\end{array}$ & $\begin{array}{c}J B 156-01, T B 3- \\
5,6,7,8 \\
\end{array}$ & $\begin{array}{c}\text { P256, 251,301 W/R } 504 \\
\text { 2-BLK, WHT, RED, SHLD } \\
\end{array}$ & \\
\hline $\begin{array}{c}J B 101-100 \text { TB4- } \\
5,6,7,8\end{array}$ & $\begin{array}{l}J B 156-01, T B 3- \\
9,10,11,12\end{array}$ & $\begin{array}{c}P 256,251,301 \text { W/R } 504 \\
3-B L K, W H T, R E D, \text { SHLD }\end{array}$ & \\
\hline $\begin{array}{c}\text { JB156-01 TB3- } \\
5,6,7,8\end{array}$ & $\begin{array}{l}\text { CONTROL CONSOLE } \\
\text { TB9-17, } 18,19,20\end{array}$ & $\begin{array}{l}P / J 156-10,-1 ; R, S, T, U \\
\text { BLK, CLR, RED, SHLD }\end{array}$ & \\
\hline $\begin{array}{l}\text { JB156-01 TB3- } \\
9,10,11,12 \\
\end{array}$ & $\begin{array}{l}\text { CONTROL CONSOLE } \\
\text { TB9-21, 22, 23, } 24 \\
\end{array}$ & $\begin{array}{l}\text { P/J156-10,-1;V,W,X,Y } \\
\text { BLK, CLR, RED, SHLD }\end{array}$ & \\
\hline TB9-17, 18 & DS13-1, PS-1+ (24VDC) & ( DS13-2 TO PS-1-) & \\
\hline TB9-21, 22 & DS14-1, PS-1+ (24VDC) & (DS14-2 TO PS-1-) & \\
\hline DS13-1 & WT-156-01-BOC & & \\
\hline DS14-1 & $W T-156-01-800$ & & \\
\hline $\begin{array}{c}\text { WT-156-01 } \\
\text { DATA, DATA* }\end{array}$ & $\begin{array}{c}P C-1 \text { RS-485 PORT } \\
T X / R X+, \quad T X / R X-\end{array}$ & TB3-2, 1 & \\
\hline
\end{tabular}

REFERENCES H-2-78948 SHT 7, 78975 SHT 384, 78981 SHT 3, 78983 SHT 1,3,4

PERFORMED BY N/A

$\operatorname{DATE}(S)$

NOT USED. 8.6 .2 SAYS TO USE ONLY IF PROBLEMS

ARE ENCOUNTERED. 


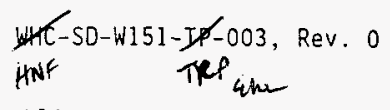

Appendix G - VibraSwitch Checklist

page 2 of 2

\begin{tabular}{|c|c|c|c|}
\hline \multicolumn{4}{|c|}{ PUMP $\# 2(P-701 A)$ VIBRASWITCH WIRING CHECK LIST } \\
\hline FROM & TO & VIA & $\checkmark$ \\
\hline $\begin{array}{l}\text { P2-VIB-1 UPPER } \\
\text { NO, COM, NC }\end{array}$ & $\begin{array}{l}\text { RTD JBOX TERMINALS } \\
14,15,16 \\
\end{array}$ & $\begin{array}{l}\text { P330 W/R } 556 \\
\text { BLK, CLR, RED } \\
\end{array}$ & \\
\hline $\begin{array}{c}\text { P2-VIB-2 LOWER } \\
\text { NO, COM, NC }\end{array}$ & $\begin{array}{l}\text { RTD JBOX TERMINALS } \\
32,33,34 \\
\end{array}$ & $\begin{array}{l}\text { P335 W/R } 567 \\
\text { BLK, CLR, RED }\end{array}$ & \\
\hline $\begin{array}{l}\text { RTD JBOX TERMINALS } \\
14,15,16 \\
\end{array}$ & $\begin{array}{l}\text { JB101-100 JB5- } \\
21,22,23,24 \\
\end{array}$ & $\begin{array}{l}\text { P329 P328 W/R } 565 \\
\text { 3-BLK, WHT, RED, SHLD }\end{array}$ & \\
\hline $\begin{array}{l}\text { RTD JBOX TERMINALS } \\
32,33,34 \\
\end{array}$ & $\begin{array}{l}\text { JB101-100 TB5- } \\
17,18,19,20 \\
\end{array}$ & $\begin{array}{l}\text { P329 P328 W/R } 565 \\
\text { 4-BLK, WHT, RED, SHLD }\end{array}$ & \\
\hline $\begin{array}{l}\text { JB101-100 TB4- } \\
21,22,23,24 \\
\end{array}$ & $\begin{array}{r}J 8156-01, T 84- \\
21,22,23,24 \\
\end{array}$ & $\begin{array}{c}\text { P256,251,301 W/R } 504 \\
\text { 9-BLK, WHT, RED, SHLD } \\
\end{array}$ & \\
\hline $\begin{array}{l}\mathrm{JB} 101-100 \mathrm{~TB} 4- \\
17,18,19,20 \\
\end{array}$ & $\begin{array}{l}\text { JB156-01 TB4- } \\
17,18,19,20 \\
\end{array}$ & $\begin{array}{c}\text { P256,251,301 W/R } 504 \\
\text { 8-BLK, WHT, RED, SHLD }\end{array}$ & \\
\hline $\begin{array}{r}J B 156-01, T B 3- \\
21,22,23,24 \\
\end{array}$ & $\begin{array}{l}\text { CONTROL CONSOLE } \\
\text { TB4-17, 18, 19, } 20 \\
\end{array}$ & $\begin{array}{l}\text { P/J156-10, }-1 ; d, \text { e, f,g } \\
\text { BLK, CLR, RED, SHLD }\end{array}$ & \\
\hline $\begin{array}{l}\text { JB156-01 TB3- } \\
17,18,19,20 \\
\end{array}$ & $\begin{array}{c}\text { CONTROL CONSOLE } \\
\text { TB4-21, } 22,23,24\end{array}$ & $\begin{array}{l}\text { P/J156-10,-1; }, \text { a , b, c } \\
\text { BLK, CLR, RED, SHLD }\end{array}$ & \\
\hline TB4-17, 18 & $D S 11-1, P S-1+(24 V D C)$ & $($ OS11-2 TO PS $-1-)$ & \\
\hline$T B 4-21,22$ & DS $12-1$, PS $-1+(24 \mathrm{VDC})$ & $($ OS12-2 TO PS-1-) & \\
\hline DS11-1 & $W T-156-01-B 0 A$ & & \\
\hline DS12-1 & $W T-156-01-B O B$ & & \\
\hline $\begin{array}{l}\text { WT-156-01 } \\
\text { DATA, DATA* }\end{array}$ & $\begin{array}{c}P C-1 \text { RS }-485 \text { PORT } \\
\text { TX/RX+, TX/RX- } \\
\end{array}$ & TB3-2, 1 & \\
\hline
\end{tabular}

REFERENCES H-2-78948 SHT 7,78975 SHT 3,78981 SHT 3, 78983 SHT 1, 3,4

PERFORMED BY

$N / A$ $\operatorname{DATE}(S)$ 


\section{WHK-SD-W151- IPS-003, Rev. 0}

Appendix H - Control Console Checklist

\begin{tabular}{|c|c|c|c|}
\hline \multicolumn{4}{|c|}{ RS-485 PUMP PADS TO PC-I WIRING CHECK LIST } \\
\hline FROM & To & VIA & $\checkmark$ \\
\hline $\begin{array}{c}\text { TX/RX+, TX/RX-, COM } \\
\text { JB100-101 } \\
\text { TB2-12,9,7 }\end{array}$ & $\begin{array}{c}\text { JB100-100 } \\
\text { TB2-15, 12, } 10\end{array}$ & $\begin{array}{l}\text { P215 / W/R } 525 \\
\text { RED, WHT, GRN }\end{array}$ & \\
\hline $\begin{array}{c}\text { TX/RX+, TX/RX-, COM, } \\
\text { SHLD -- JB100-100 } \\
\text { TB-15, 12, 10, } 16 \\
\end{array}$ & $\begin{array}{c}\text { JB156-0I }(156 \mathrm{BLDG}) \\
\mathrm{TB3}-1,2,3,4\end{array}$ & $\begin{array}{l}\text { P206,261,301 / WR504 } \\
\text { 1-RED, BLK, WHT, SHLD }\end{array}$ & \\
\hline $\begin{array}{l}\text { JB156-OI } \\
\text { TB3-1, 2, 3, } 4\end{array}$ & $\begin{array}{c}\text { CONTROL CONSOLE } \\
\text { TB3-13,14,15, } 16 \\
\end{array}$ & $\begin{array}{l}\text { P/J156-10 N,O,P,Q; } \\
\text { P/J156-1 N,O,P,Q; } \\
\text { BLK, RED, CLR, SHLD }\end{array}$ & \\
\hline TB3-13,14, 1516 & & JUMPERS & \\
\hline TB3-1, 2, 3 & $\begin{array}{c}\text { PC RS485 PORT } 1 \\
\text { TX/RX+, TX/RX-, COM } \\
\end{array}$ & $\begin{array}{l}\text { BLK, RED, SHLD TO } \\
\text { PINS } 184,283,5 \\
\end{array}$ & \\
\hline
\end{tabular}

REFERENCES H-2-78975 SHT 3-4. H-2-78981 SHT 3, H-2-78983 SHT 3-4, H-2-78948 SHT 2, 3 , AND 10. AND VENDOR DATA
PERFORMED BX
$\operatorname{DATE}(S)+1 / 97$ 
WhtC-SD-W151-IP-003, Rev. 0

Appendix I - Motor Bearing Temp Checklist

page 1 of 2

\begin{tabular}{|c|c|c|c|}
\hline \multicolumn{4}{|c|}{ PUMP \#1 ( P-702A) MOTOR BEARING TEMPERATURE WIRING CHECK LIST } \\
\hline FROM & TO & VIA & $\checkmark$ \\
\hline $\begin{array}{l}\text { LOWER BEARING RTD } \\
\text { RED, BLK, WHT }\end{array}$ & $\begin{array}{l}\text { RTD JBOX TERMINALS } \\
10,11,12\end{array}$ & & \\
\hline $\begin{array}{l}\text { UPPER BEARING RTD } \\
\text { RED, BLK, WHT }\end{array}$ & $\begin{array}{l}\text { RTD JBOX TERMINALS } \\
28,29,30 \\
\end{array}$ & & \\
\hline $\begin{array}{l}\text { RTD JBOX TERMINALS } \\
10,11,12\end{array}$ & $\begin{aligned} & \text { JB101-101 } \text { WT-I01-19 } \\
& \text { +SENSE } /+\mathrm{I}, \text {-SENSE, } \\
&\end{aligned}$ & $\begin{array}{l}\text { P333, P332, W/R } 560 \\
\text { l-RED, l-BLK, l-WHT }\end{array}$ & \\
\hline $\begin{array}{l}\text { RTD JBOX TERMINALS } \\
28,29,30\end{array}$ & $\begin{aligned} \text { JB101-101 } & \text { WT-101-20 } \\
+ \text { +SENSE } /+\mathrm{I}, & \text {-SENSE, -I }\end{aligned}$ & $\begin{array}{l}\text { P333, P332, W/R } 560 \\
2-R E D, 2-B L K, 2-W H T\end{array}$ & \\
\hline $\begin{array}{c}\text { WT-101-19 } \\
\text { DATA, DATA*, GND }\end{array}$ & $\begin{array}{c}\text { TB2-12, } 9,7 \\
\text { TXRX+, TXRX-, GND } \\
\end{array}$ & RED, WHT, GRN & \\
\hline $\begin{array}{c}\text { WT-101-20 } \\
\text { DATA, DATA*, GND }\end{array}$ & $\begin{array}{c}\text { TB2-12, 9, } 7 \\
\text { TXRX+, TXRX-, GND }\end{array}$ & RED, WHT, GRN & \\
\hline
\end{tabular}

REFERENCES H-2-78948 SHT 7, H-2-78975 SHT 4

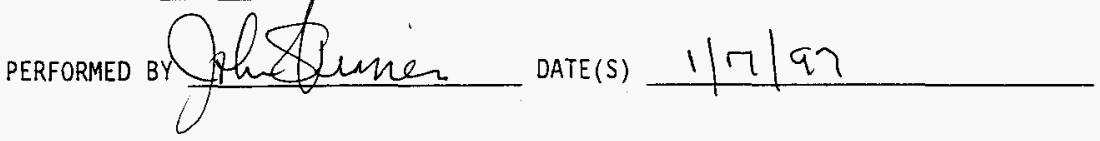


WHTC-SD-W151-IDP-003, Rev. 0
WNE TRP Gim

Appendix I - Motor Bearing Temp CheckTist page 2 of 2

\begin{tabular}{|c|c|c|c|}
\hline \multicolumn{4}{|c|}{ PUMP \#2 ( P-701A) MOTOR BEARING TEMPERATURE WIRING CHECK LIST } \\
\hline FROM & TO & VIA & $\checkmark$ \\
\hline $\begin{array}{l}\text { LOWER BEARING RTD } \\
\text { RED, BLK, WHT }\end{array}$ & $\begin{array}{l}\text { RTD JBOX TERMINALS } \\
10,11,12\end{array}$ & & \\
\hline $\begin{array}{l}\text { UPPER BEARING RTD } \\
\text { RED, BLK, WHT }\end{array}$ & $\begin{array}{c}\text { RTD JBOX TERMINALS } \\
28,29,30 \\
\end{array}$ & & \\
\hline $\begin{array}{c}\text { RTD JBOX TERMINALS } \\
10,11,12 \\
\end{array}$ & $\begin{array}{r}\text { JB101-100 WT-101-17 } \\
+ \text { +SENSE } /+1,- \text { SENSE, -I } \\
\end{array}$ & $\begin{array}{l}\text { P329, P328, W/R } 565 \\
1-R E D, \quad 1-B L K, \quad 1-W H T\end{array}$ & \\
\hline $\begin{array}{l}\text { RTD JBOX TERMINALS } \\
28,29,30\end{array}$ & $\begin{aligned} \text { JBI01-100 } & \text { WT-101-18 } \\
+ \text { SENSE }+I, & - \text { SENSE, }-I\end{aligned}$ & $\begin{array}{l}\text { P329, P328, W/R } 565 \\
\text { 2-RED, 2-BLK, 2-WHT }\end{array}$ & \\
\hline $\begin{array}{c}\text { WT-101-17 } \\
\text { DATA, DATA*, GND }\end{array}$ & $\begin{array}{r}\text { TB2-15, 12, } 10 \\
\text { TXRX+, TXRX-, GND } \\
\end{array}$ & RED, WHT, GRN & \\
\hline $\begin{array}{c}\text { WT-101-18 } \\
\text { DATA, DATA*, GND }\end{array}$ & $\begin{array}{r}\text { TB2-15, 12, } 10 \\
\text { TXRX+, TXRX-, GND } \\
\end{array}$ & RED, WHT, GRN & \\
\hline
\end{tabular}

REFERENCES $\frac{\mathrm{H}-2-78948 \text {, SHT 7. H-2-78975 SHT 3 }}{\text { DATE(S) } 1 / 7 / 97}$ 
Wht-SD-W151-DP-003, Rev. 0
HNS TRe
and

Appendix J - Strain Gage Checklist

\begin{tabular}{|c|c|c|c|}
\hline \multicolumn{4}{|c|}{ PROBE I5E STRAIN GAUGE WIRING CHECK LIST } \\
\hline FROM & TO & VIA & $\checkmark$ \\
\hline $\begin{array}{c}\text { WE-701A }\left(-3 \mathrm{FT}+60^{\circ}\right) \\
\text { BLK, YEL, BRN }\end{array}$ & TB3 $-2,3,4$ & STRAIN GAUGE LEADS & \\
\hline $\begin{array}{c}\text { WE-702A }\left(-20 \mathrm{FT}+45^{\circ}\right) \\
\text { BLK, YEL, BRN }\end{array}$ & TB3-9, 10,11 & STRAIN GAUGE LEADS & \\
\hline $\begin{array}{c}\text { WE-703A }\left(-32 \mathrm{FT}+30^{\circ}\right) \\
\text { BLK, YEL, BRN }\end{array}$ & TB3-16, 17,18 & STRAIN GAUGE LEADS & \\
\hline $\begin{array}{r}\text { WE-704A }\left(-3 \mathrm{FT}-60^{\circ}\right) \\
\text { BLK, YEL, BRN }\end{array}$ & $T B 2-2,3,4$ & STRAIN GAUGE LEADS & \\
\hline $\begin{array}{c}\text { WE-705A }\left(-20 \mathrm{FT}-45^{\circ}\right) \\
\text { BLK, YEL, BRN }\end{array}$ & $T B 2-9,10,11$ & STRAIN GAUGE LEADS & \\
\hline $\begin{array}{c}\text { WE-706A }\left(-32 \mathrm{FT}-30^{\circ}\right) \\
\text { BLK, YEL, BRN }\end{array}$ & $T B 2-16,17,18$ & STRAIN GAUGE LEADS & \\
\hline TB3-2, $4,5,6$ & $\begin{array}{r}\text { WT-101-1 ( } A) \\
+I N,-E X C,+E X C,-I N \\
\end{array}$ & RED, ORN, BRN, WHT & \\
\hline TB3-9, 11, 12, 13 & $\begin{array}{r}W T-101-2(\mathrm{~A}) \\
+I N,-E X C,+E X C,-I N \\
\end{array}$ & RED, ORN, BRN, WHT & \\
\hline$T B 3-16,18,19,20$ & $\begin{array}{r}W T-101-3(A) \\
+I N,-E X C,+E X C,-I N \\
\end{array}$ & RED, ORN, BRN, WHT & \\
\hline TB2-2, 4, 5, 6 & $\begin{aligned} & W T-101-4(A) \\
&+ I N,-E X C,+E X C,-I N \\
&\end{aligned}$ & RED, ORN, BRN, WHT & \\
\hline $\mathrm{TB} 2-9,11,12,13$ & $\begin{aligned} & W T-10 I-5(A) \\
&+ I N,-E X C,+E X C,-I N . \\
&\end{aligned}$ & RED, ORN, BRN, WHT & \\
\hline TB2-16, 18, 19, 20 & $\begin{aligned} & W T-101-6(A) \\
&+ I N,-E X C,+E X C,-I N \\
&\end{aligned}$ & RED, ORN, BRN, WHT & \\
\hline $\begin{array}{l}\text { WT-101-1 THRU } 6 \\
\text { DATA, DATA*, +VS, GND }\end{array}$ & $\begin{array}{c}\text { TB1-1, } 4,7,8 \\
\text { TXRX+, TXRX- }+24, \text { GND }\end{array}$ & YEL, GRN, BLU, BLK & \\
\hline $\mathrm{TB} 1-1,8,4,8,7,8$ & $\begin{array}{c}\text { JB101-101 TB2- } \\
12,13,9,10,15,16\end{array}$ & $\begin{array}{l}\text { W/R } 526 \text { RED/BLK, } \\
\text { WHT/BLK, BLK/GRN } \\
\end{array}$ & \\
\hline
\end{tabular}

REFERENCES $\mathrm{H}-2-78977$ SHT 3, H-2-78975 SHT 4, H-2-78948 SHT 2, VENDOR DATA

PERFORMED BY

$\operatorname{DATE}(S)$ 
WWK-SD-W151- IP-003, Rev. 0
HWF RP

Appendix J - Strain Gage Checklist

\begin{tabular}{|c|c|c|c|}
\hline \multicolumn{4}{|c|}{ PROBE $15 F$ STRAIN GAUGE WIRING CHECK LIST } \\
\hline FROM & To & VIA & $\sqrt{2}$ \\
\hline $\begin{array}{c}\text { WE-707A }\left(-3 \mathrm{FT}+60^{\circ}\right) \\
\text { BLK, YEL, BRN }\end{array}$ & TB3-2, 3,4 & STRAIN GAUGE LEADS & \\
\hline 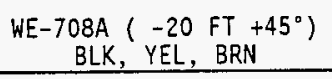 & $\mathrm{TB} 3-9,10,11$ & STRAIN GAUGE LEADS & \\
\hline $\begin{array}{c}\text { WE-709A }\left(-32 \mathrm{FT}+30^{\circ}\right) \\
\text { BLK, YEL, BRN }\end{array}$ & TB3-16, 17,18 & STRAIN GAUGE LEADS & \\
\hline $\begin{array}{c}\text { WE-710A }\left(-3 \text { FT }-60^{\circ}\right) \\
\text { BLK, YEL, BRN }\end{array}$ & TB2-2, 3,4 & STRAIN GAUGE LEADS & \\
\hline $\begin{array}{c}\text { WE-71IA }\left(-20 \text { FT }-45^{\circ}\right) \\
\text { BLK, YEL, BRN }\end{array}$ & $\mathrm{TB} 2-9,10,11$ & STRAIN GAUGE LEADS & \\
\hline 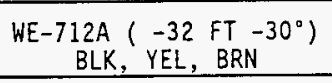 & TB2-16, 17,18 & STRAIN GAUGE LEADS & \\
\hline TB3-2, 4, 5, 6 & $\begin{array}{c}\text { WT-101-1 ( } A) \\
+ \text { IN, }-E X C,+E X C,-I N\end{array}$ & RED, ORN, BRN, WHT & \\
\hline TB3-9, $11,12,13$ & $\begin{aligned} & W T-101-2(A) \\
&+ I N,-E X C,+E X C,-I N \\
&\end{aligned}$ & RED, ORN, BRN, WHT & \\
\hline TB3-16, 18, 19, 20 & $\begin{array}{c}\text { WT-101-3 (A) } \\
+ \text { IN, - EXC, +EXC, -IN } \\
\end{array}$ & RED, ORN, BRN, WHT & \\
\hline TB2-2, 4, 5, 6 & $\begin{aligned} & W T-101-4(A) \\
&+ I N,-E X C,+E X C,-I N \\
&\end{aligned}$ & RED, ORN, BRN, WHT & \\
\hline TB2-9, $11,12,13$ & $\begin{aligned} & \text { WT-101-5 ( A ) } \\
&+ I N,-E X C,+E X C,-I N \\
&\end{aligned}$ & RED, ORN, BRN, WHT & \\
\hline TB2-16, 18, 19, 20 & $\begin{aligned} & W T-101-6(A) \\
&+ I N,-E X C,+E X C,-I N \\
&\end{aligned}$ & RED, ORN, BRN, WHT & \\
\hline $\begin{array}{c}\text { WT-101-1 THRU } 6 \\
\text { DATA, DATA*, +VS, GND }\end{array}$ & $\begin{array}{c}\text { TB1-1, 4, 7, } 8 \\
\text { TXRX+, TXRX-,+24, GND }\end{array}$ & YEL, GRN, BLU, BLK & \\
\hline$T B 1-1,8,4,8,7,8$ & $\begin{array}{c}\text { JB101-101 TB2- } \\
12,13,9,10,18,19 \\
\end{array}$ & $\begin{array}{l}\text { W/R } 529 \text { RED/BLK, } \\
\text { WHT/BLK, BLK/GRN } \\
\end{array}$ & \\
\hline
\end{tabular}

REFERENCES H-2-78978 SHT 3, H-2-78975 SHT 4, H-2-78948 SHT 2, VENDOR DATA PERFORMED BY DATE(S) 
WhC-SD-W151-IF-003, Rev. 0
itht TRe

Appendix K - Sludge Mobilization Cart Checklist

page 1 of 2

\begin{tabular}{|c|c|c|c|}
\hline \multicolumn{4}{|c|}{ SLUDGE MOBILIZATION GAMMA DATA WIRING CHECK LIST - CART \#1 (\#2) } \\
\hline FROM & To & VIA & $\checkmark$ \\
\hline AMPLIFIER DEAD TIME & $\begin{array}{l}\text { DST-001 ANALOG IN } \\
\text { ADDRESS () }\end{array}$ & COAX CABLE & \\
\hline COUNT RATEMETER & $\begin{array}{l}\text { DST-002 ANALOG IN } \\
\text { ADDRESS () }\end{array}$ & COAX CABLE & \\
\hline $\begin{array}{l}\text { MT-001 TBC-8, } 9,3 \\
\text { ADDRESS () }\end{array}$ & $T X / R X+, T X / R X-$, COMM & RS-485 BUS & \\
\hline $\begin{array}{l}\text { DST-001 DATA, DATA*, } \\
\text { +VS, GND }\end{array}$ & $\begin{array}{c}T X / R X+, T^{T X / R X-,}+V S, \\
\text { COMM }\end{array}$ & RS-485 BUS & \\
\hline $\begin{array}{l}\text { DST-002 DATA, DATA*, } \\
\text { +VS, GND }\end{array}$ & $\begin{array}{c}T X / R X+, T X / R X-,+V S, \\
\text { COMM }\end{array}$ & RS-485 BUS & \\
\hline$T X / R X+, \underset{C O M M}{T X / R X-,+V S,}$ & TB2-1, 2, 3, 4 & RS485 BUS & \\
\hline TB2-1, $2,3,4$ & $\mathrm{~J} 2-\mathrm{A}, \mathrm{B}, \mathrm{C}, \mathrm{O}$ & JUMPERS & \\
\hline$J 2-A, B, C, D$ & $\begin{array}{c}J B 100-100 \text { OR }-101 \\
\text { J1-A, B, C, D }\end{array}$ & UMBILICAL CABLE & \\
\hline $\begin{array}{c}J B 100-100 \text { OR }-101 \\
J 1-A, B, C, D\end{array}$ & TB2-15, 12, 17, 16 & & \\
\hline
\end{tabular}

REFERENCES H-2-79233 SHT 1-2, H-2-78944, H-2-78975 SHT 3-4, VENDOR DATA

PERFORMED BY

$\operatorname{DATE}(S)$ 
WhC-SD-W151-IP-003, Rev. 0 HN ThP.

Appendix L - Instrument Check List

cint

\begin{tabular}{|c|c|c|c|c|c|}
\hline $\begin{array}{l}\text { INSTR. TYPE } \\
\text { \& IDENT. }\end{array}$ & $\begin{array}{l}\text { NOMINAL } \\
\text { INPUT } \\
\end{array}$ & $\begin{array}{l}\text { ACTUAL } \\
\text { INPUT } \\
\end{array}$ & $\begin{array}{l}\text { NOMINAL } \\
\text { RESPONSE } \\
\end{array}$ & $\begin{array}{c}\text { ACTUAL } \\
\text { RESPONSE } \\
\end{array}$ & $\checkmark$ \\
\hline $\begin{array}{c}\text { NEWPORT - DM2 } \\
\text { PUMP ORIENT } \\
\text { PUIMP } 1\end{array}$ & $\begin{array}{r}-5.00 \mathrm{VDC} \\
0.00 \mathrm{VDC} \\
+5.00 \mathrm{VDC} \\
\end{array}$ & & $\begin{array}{r}-90.0 \\
0.0 \\
+90.0 \\
\end{array}$ & & \\
\hline $\begin{array}{l}\text { NEWPORT - DM2 } \\
\text { PUMP ORIENT } \\
\text { PUIP } 2 \\
\end{array}$ & $\begin{array}{c}-5.00 \mathrm{VDC} \\
0.00 \mathrm{VDC} \\
+5.00 \mathrm{VDC} \\
\end{array}$ & & $\begin{array}{r}-90.0 \\
0.0 \\
+90.0 \\
\end{array}$ & & \\
\hline $\begin{array}{l}\text { NEWPORT - DM4 } \\
\text { PUMP } 1 \text { AMPS }\end{array}$ & $\begin{array}{c}0.00 \mathrm{VDC} \\
10.00 \mathrm{VDC}\end{array}$ & & $\begin{array}{r}0.0 \\
365.0 \\
\end{array}$ & & \\
\hline $\begin{array}{l}\text { NENPORT - DM5 } \\
\text { PUMP } 1 \text { VOLTS }\end{array}$ & $\begin{array}{c}0.00 \mathrm{VDC} \\
10.00 \mathrm{VDC} \\
\end{array}$ & & $\begin{array}{r}0.0 \\
480.0 \\
\end{array}$ & & \\
\hline $\begin{array}{l}\text { NEWPORT - DM6 } \\
\text { PUMP I FREQ. }\end{array}$ & $\begin{array}{c}0.00 \mathrm{VDC} \\
10.00 \mathrm{VDC}\end{array}$ & & $\begin{array}{c}0.0-60.0 \\
\text { OR } \\
0.0-1180.0 \\
\end{array}$ & & \\
\hline $\begin{array}{l}\text { NEWPORT - DM10 } \\
\text { PUMP I AMPS }\end{array}$ & $\begin{array}{c}0.00 \mathrm{VDC} \\
10.00 \mathrm{VDC} \\
\end{array}$ & & $\begin{array}{r}0.0 \\
365.0 \\
\end{array}$ & & \\
\hline $\begin{array}{l}\text { NEWPORT - DMI } 1 \\
\text { PUMP } 1 \text { VOLTS }\end{array}$ & $\begin{array}{c}0.00 \mathrm{VDC} \\
10.00 \mathrm{VDC} \\
\end{array}$ & & $\begin{array}{r}0.0 \\
480.0 \\
\end{array}$ & & \\
\hline $\begin{array}{l}\text { NEWPORT - DMI2 } \\
\text { PUMP } 1 \text { FREQ. }\end{array}$ & $\begin{array}{c}0.00 \mathrm{VDC} \\
10.00 \mathrm{VDC}\end{array}$ & & $\begin{array}{c}0.0-60.0 \\
O R \\
0.0-1180.0 \\
\end{array}$ & & \\
\hline $\begin{array}{c}\text { OMEGA } 1412 \\
\text { WT-101-17 } \\
\text { PMP } 2 \text { BOT BRG }\end{array}$ & 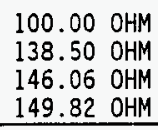 & & 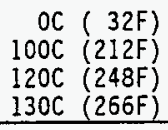 & & \\
\hline $\begin{array}{l}\text { OMEGA } 1412 \\
\text { WT-101-18 } \\
\text { PMP } 2 \text { TOP BRG }\end{array}$ & 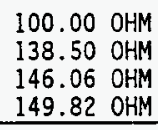 & & $\begin{array}{r}0 \mathrm{C}(32 \mathrm{~F}) \\
100 \mathrm{C}(212 \mathrm{~F}) \\
120 \mathrm{C}(248 \mathrm{~F}) \\
130 \mathrm{C}(266 \mathrm{~F}) \\
\end{array}$ & & \\
\hline $\begin{array}{l}\text { OMEGA } 1412 \\
\text { WT-101-19 } \\
\text { PMP } 1 \text { BOT BRG }\end{array}$ & $\begin{array}{l}100.00 \mathrm{OHM} \\
138.50 \mathrm{OHM} \\
146.06 \mathrm{OHM} \\
149.82 \mathrm{OHM} \\
\end{array}$ & & $\begin{array}{r}0 \mathrm{C}(32 \mathrm{~F}) \\
100 \mathrm{C}(212 \mathrm{~F}) \\
120 \mathrm{C}(248 \mathrm{~F}) \\
130 \mathrm{C}(266 \mathrm{~F}) \\
\end{array}$ & & \\
\hline $\begin{array}{c}\text { OMEGA } 1412 \\
\text { WT-101-20 } \\
\text { PMP I TOP BRG }\end{array}$ & 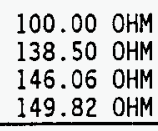 & & $\begin{array}{r}0 \mathrm{C}(32 \mathrm{~F}) \\
100 \mathrm{C}(212 \mathrm{~F}) \\
120 \mathrm{C}(248 \mathrm{~F}) \\
130 \mathrm{C}(266 \mathrm{~F})\end{array}$ & & \\
\hline $\begin{array}{l}\text { OMEGA } 1142 \\
\text { WT-DST-OO1 } \\
\text { CART I BUSY }\end{array}$ & $\begin{array}{l}0.3 \mathrm{VDC} \\
5.0 \mathrm{VDC}\end{array}$ & & $\begin{array}{r}0 \% \\
100 \% \\
\text { BUSY } \\
\end{array}$ & & \\
\hline
\end{tabular}




\section{WHC-SD-W151-IP-003, Rev. 0 itwF TRE cunt}

\begin{tabular}{|c|c|c|c|c|c|}
\hline $\begin{array}{l}\text { INSTR. TYPE } \\
\& \text { IDENT. }\end{array}$ & $\begin{array}{c}\text { NOMINAL } \\
\text { INPUT } \\
\end{array}$ & $\begin{array}{l}\text { ACTUAL } \\
\text { INPUT } \\
\end{array}$ & $\begin{array}{l}\text { NOMINAL } \\
\text { RESPONSE } \\
\end{array}$ & $\begin{array}{c}\text { ACTUAL } \\
\text { RESPONSE } \\
\end{array}$ & $\checkmark$ \\
\hline $\begin{array}{l}\text { OMEGA } 1142 \\
\text { WT-DST-002 } \\
\text { CART } 1 \text { COUNT }\end{array}$ & $\begin{array}{c}0.00 \mathrm{VDC} \\
10.00 \mathrm{VDC}\end{array}$ & & FULL $\stackrel{0}{\text { SCALE }}$ & & \\
\hline $\begin{array}{l}\text { RED LION LGD } \\
\text { WT-MT-001 } \\
\text { CART I FOOTAGE }\end{array}$ & $\begin{array}{l}\text { RAISE \& LOWER } \\
\text { PROBE -- } \\
\text { MEASURE POS. }\end{array}$ & & $\begin{array}{l}\text { SAME AS RED } \\
\text { LION LGD \& } \\
\text { MEASURED }\end{array}$ & & \\
\hline $\begin{array}{l}\text { OMEGA } 1142 \\
\text { WT-DST-003 } \\
\text { CART } 2 \text { BUSY } \\
\end{array}$ & $\begin{array}{l}0.3 \mathrm{VDC} \\
5.0 \mathrm{VDC}\end{array}$ & & $\begin{array}{r}0 \% \\
100 \% \\
\text { BUSY } \\
\end{array}$ & & \\
\hline $\begin{array}{l}\text { OMEGA } 1142 \\
\text { WT-DST-004 } \\
\text { CART } 2 \text { COUNT }\end{array}$ & $\begin{array}{c}0.00 \mathrm{VDC} \\
10.00 \mathrm{VDC}\end{array}$ & & FULL $\stackrel{0}{\text { SCALE }}$ & & \\
\hline $\begin{array}{l}\text { RED LION LGD } \\
\text { WT-MT-001 } \\
\text { CART } 2 \text { FOOTAGE }\end{array}$ & $\begin{array}{c}\text { RAISE \& } \\
\text { LOWER } \\
\text { PROBE } \\
\end{array}$ & & $\begin{array}{l}\text { SAME AS RED } \\
\text { LION LGD } \\
\text { DISPLAY }\end{array}$ & & \\
\hline $\begin{array}{l}\text { STRAIN GAGES } \\
\text { RISER 15E }\end{array}$ & $\begin{aligned} & \text { SWITCH IN } \\
& \approx 350 K \Omega \text { CAL } \\
& \text { RESISTOR } \\
&\end{aligned}$ & & $\begin{array}{l}\approx 2.5 \mathrm{mV}, \\
\approx 500 \mu \mathrm{\mu}, \\
\approx 6001 \mathrm{bf}\end{array}$ & & \\
\hline $\begin{array}{l}\text { STRAIN GAGES } \\
\text { RISER } 15 \mathrm{~F}\end{array}$ & $\begin{array}{r}\text { SWITCH IN } \\
\approx 350 K \Omega \text { CAL } \\
\text { RESISTOR } \\
\end{array}$ & & $\begin{array}{l}\approx 2.5 \mathrm{mV}, \\
\approx 500 \mu \epsilon, \\
\approx 6001 \mathrm{bf}\end{array}$ & & \\
\hline
\end{tabular}

\section{REFERENCES}

PERFORMED BY $\operatorname{DATE}(S)$ 
Project Title/Work Order:

Project $W-151$, Tank 101-AZ Waste Retrieval System

Checkout-Testing Report, HNF-SD-W151-TRP-003

EDT No.: 610579

ECN No.: $\quad N / A$

charge code: D2D1A

\begin{tabular}{|c|c|c|c|c|}
\hline Name & MSIN & $\begin{array}{c}\text { with } \\
\text { Attachment }\end{array}$ & $\begin{array}{c}\text { EDT/ECN \& } \\
\text { Comment }\end{array}$ & $\begin{array}{c}\text { EDT/ECN } \\
\text { Only }\end{array}$ \\
\hline $\begin{array}{l}\text { E. F. Enloe } \\
\text { Central Files } \\
\text { Project Files }\end{array}$ & $\begin{array}{l}S 2-56 \\
A 3-88 \\
R 1-29\end{array}$ & $\begin{array}{l}x \\
x \\
x\end{array}$ & & \\
\hline
\end{tabular}

